\title{
Dynamic Volume-Return Relation of Individual Stocks*
}

\author{
Guillermo Llorente Roni Michaely Gideon Saar Jiang Wang
}

First version: November 15, 1997

Current version: August 15, 2000

\begin{abstract}
We examine the dynamic relation between return and volume of individual stocks. Using a simple model in which investors trade to share risk or speculate on private information, we show that returns generated by risk-sharing trades tend to reverse themselves while returns generated by speculative trades tend to continue themselves. We test this theoretical prediction by analyzing the relation between daily volume and first-order return autocorrelation for individual stocks listed on the NYSE and AMEX. We find that the cross-sectional variation in the relation between volume and return autocorrelation is related to the extent of informed trading in a manner consistent with the theoretical prediction.
\end{abstract}

${ }^{*}$ Llorente is from Facultad de C. Economicas, Universidad Autonoma de Madrid, 28049 Madrid, Spain (telephone: 34-91-397-4812, fax: 34-91-397-4091, and email: guiller@uam.es); Michaely is from the Johnson School of Management, Cornell University, Ithaca, NY 14850 (telephone: 607-255-7209, fax: 607254-4590, and email: rm34@cornell .edu); Saar is from the Stern School of Business, New York University, New York, NY 10012 (telephone: 212-998-0318, fax: 212-995-4233, and email: gsaar@stern.nyu.edu); Wang is from the Sloan School of Management, MIT, Cambridge, MA 02142-1347 and the National Bureau of Economic Research (telephone: 617-253-2632, fax: 617-258-6855, and email: wangj@mit.edu). The authors thank I/B/E/S for providing the data on analysts' earnings forecasts, Yiming Qian for her research assistance, and Juan del Hoyo, David Easley, Gustavo Grullon, John Heaton, Soeren Hvidkjaer, Robert Jarrow, Andrew Lo, N. Nimalendran, Maureen O'Hara, Bhaskaran Swaminathan, seminar participants at Cornell University, New York University, the 1998 Western Finance Association Meetings, and a referee for helpful comments. 


\section{Introduction}

Market participants carefully watch the volume of trade, which presumably conveys valuable information about future price movements. At the heart of the matter are the questions of why investors trade and how trades with different motives relate to prices. Two reasons are often mentioned for why investors trade: to rebalance their portfolios for risk sharing and to speculate on their private information. These two types of trades, which we call hedging and speculative trades, respectively, result in different return dynamics.

For example, when a subset of investors sells a stock for hedging reasons, the stock's price must decrease to attract other investors to buy. Since the expectation of future stock payoff remains the same, the decrease in the price causes a low return in the current period and a high expected return for the next period. However, when a subset of investors sells a stock for speculative reasons, its price decreases, reflecting the negative private information about its future payoff. Since this information is usually only partially impounded into the price, the low return in the current period will be followed by a low return in the next period, when the negative private information is further reflected in the price. This example shows that hedging trades generate negatively autocorrelated returns and speculative trades generate positively autocorrelated returns.

Intensive trading volume can help to identify the periods in which either allocational or informational shocks occur, and thus can provide valuable information to market observers about future price movements of the stock. In periods of high volume, stocks with a high degree of speculative trading tend to exhibit positive return autocorrelation and stocks with a low degree of speculative trading tend to exhibit negative return autocorrelation.

In this paper, we construct a simple equilibrium model to derive the return dynamics generated when investors trade both to hedge and to speculate. The model illustrates that the relation of current return, volume, and future returns depends on the relative significance of speculative trade versus hedging trade. If speculative trading in a stock is relatively insignificant, returns accompanied by high volume tend to reverse themselves in the subsequent period. If speculative trading in a stock is significant, conditioned on high volume, returns become less likely to reverse and can even continue in the subsequent period. The difference in the relative importance of speculative trading among different stocks gives rise to the cross-sectional variation in their volume-return dynamics.

We empirically test the predictions of the model by analyzing the daily volume-return dynamics of individual stocks traded on the NYSE and AMEX. The basic structure of the empirical tests is as follows. For each stock in the sample, we use a time-series regression 
to find the relation between current return and volume and future return. Then, guided by the predictions of the model, we examine how this relation varies across stocks with the extent of speculative trading. We consider several proxies, such as market capitalization and bid-ask spread, for the degree of speculative trading across stocks. Consistent with the model, we find significant differences in the dynamics of returns and volume across stocks with different degrees of information asymmetry. Stocks of smaller firms, or stocks with higher bid-ask spreads, show a tendency for return continuation following high volume days. Stocks of larger firms, or stocks with smaller bid-ask spreads, show almost no continuation and mostly return reversal following high-volume days.

We examine the robustness of our results along several dimensions. First, we explore alternative econometric specifications of our tests and find that they do not change our results. Second, we replace the daily interval with a measurement period that equates the amount of noise trading across stocks, and show that it does not affect our findings. Third, we check the sensitivity of our findings to the effect of the bid-ask bounce, which can also cause return autocorrelation. We perform this check by repeating our main experiment using returns calculated from mid-quotes rather than from the closing price of the day. This alternative procedure does not alter our conclusions. Fourth, we show that our findings are not sensitive to alternative definitions of trading volume. Fifth, in light of recent papers that identified a larger analyst following with a smaller adverse selection problem, we use analyst following as an additional proxy for information asymmetry. Our results show that stocks that are followed by more analysts exhibit less return continuation following high volume days.

We also test the hypothesis that it is firm-specific private information that affects the cross-sectional variation in the dynamic volume-return relation. We decompose both the volume and return series into systematic and unsystematic components. We find that the relation between information asymmetry and the influence of volume on the autocorrelation of returns persists when we remove the market-wide variations from the analysis.

Many recent papers investigate the relation between return dynamics and trading volume. Several of them focus on aggregate returns and volume (e.g., Campbell, Grossman, and Wang, 1993 (henceforth CGW); Duffee, 1992; Gallant, Rossi, and Tauchen, 1992; and LeBaron, 1992). These studies find that returns on high-volume days tend to reverse themselves.

A few studies also use returns and volume of individual stocks (e.g., Antoniewicz, 1993; 
Conrad, Hameed, and Niden, 1992; Morse, 1980; Stickel and Verrecchia, 1994). In particular, Antoniewicz finds that returns of individual stocks on high-volume days are more sustainable than are returns on low-volume days. Stickel and Verrecchia find that when earnings announcements are accompanied by higher volume, returns are more sustainable in the following days. The results of these two papers, from pooling together individual returns and volume, contrasts with the result from aggregate returns and volume. However, these studies do not provide an explanation that reconciles the two phenomena, nor do they examine the cross-sectional variation in the volume-return relation among the stocks. $^{1}$

Our paper provides a model that reconciles the contrasting empirical results on the volume-return relation at the aggregate and the individual levels. The model demonstrates how these results are related to the cross-sectional variation in the volume-return relation. Unlike CGW, we recognize information asymmetry as an important trading motive in addition to risk sharing. We show that for stocks with low information asymmetry (like the market indices and the big firms studied by CGW and LeBaron, 1992), returns following high-volume days exhibit strong reversals, as in CGW. However, for stocks with high information asymmetry, returns following high-volume days exhibit only weaker reversals or even continuations, which is consistent with the findings of Antoniewicz (1993) and Stickel and Verrecchia (1994) who use pooled returns and volume of individual stocks. Our analysis highlights the importance of information asymmetry in understanding the dynamic volume-return relation and demonstrates the more general nature of this relation, which was only partially captured by the aforementioned papers.

On the theoretical side, our model is very close to that of Wang (1994) but less complex. Simplification allows us to obtain sharper predictions on the dependence of a stock's dynamic volume-return relation on the extent of information asymmetry, which we test empirically. Our paper is also related to a paper by Blume, Easley, and O'Hara (1994), which examines the informational role of volume. In their model, investors can extract useful information from both volume and prices. In our model, volume provides no additional information to investors. However, the dynamic relation between volume and returns allows observers of the economy to better understand its underlying characteris-

\footnotetext{
${ }^{1}$ In a recent paper, Lee and Swaminathan (2000) show that past volume provides valuable information about future returns over horizons of six months. By assigning stocks to portfolios based on past volume and price changes, they show that using the prior six-months-volume in combination with price changes is superior to using past price changes alone in predicting long term returns. Specifically, they show that buying low-volume winners and selling past high-volume losers outperform a pure price momentum strategy. The nature of their result is similar to that of Antoniewicz (1993), but at a longer horizon.
} 
tics, such as the degree of information asymmetry, which is our focus.

Other related papers include Brown and Jennings (1989) and Grundy and McNichols (1989). In particular, Brown and Jennings examine the characteristics of return autocorrelations when investors trade on private information. They do not consider volume, which is partially exogenous in their model due to the presence of noise traders. We focus on the joint behavior of return and volume because it provides more information about the underlying economy. ${ }^{2}$

On the empirical side, our paper is related to Hasbrouck $(1988,1991)$ who utilizes transactions data to examine the impact of trades on prices and quotes. He uses a linear empirical model to capture how such an impact might be related to the inventory control of specialists and to the private information behind trades. Even though we do not focus on the actual trading process, in many ways our paper deals with the same issues, namely, how trades with different motives generate different return dynamics. Nonetheless, our paper is different in several ways. First, while Hasbrouck's analysis is based on heuristic linear specifications, our analysis is based on a theoretical model, which leads to a particular non-linear specification. Second, Hasbrouck's analysis conditions on a trade's direction, relying on a heuristic algorithm to infer its direction from publically available data. We prefer to use volume that is not subject to errors introduced by trade classification algorithms. Third, our analysis focuses on the cross-sectional difference among stocks.

The paper proceeds as follows. Section 2 presents the model and the theoretical predictions that we test. Section 3 describes the empirical methodology and Section 4 provides our empirical results. Section 6 concludes. All proofs are contained in the Appendix.

\section{The Model}

In this section, we present a model of the stock market in which investors trade for both allocation and information reasons. We use the model to show how the dynamic relation between return and volume depends on the information asymmetry between investors. Since our goal is to establish this dependence and to illustrate the economic forces behind

\footnotetext{
${ }^{2}$ It should also be noted that many theoretical papers in market microstructure deal with the impact of private information on asset prices (e.g., Easley and O'Hara, 1987; Glosten and Milgrom, 1985; Kyle, 1985). However, the assumption of a competitive market maker in these models makes prices follow a martingale, eliminating richer dynamics.
} 
it, we keep the model as parsimonious as possible. We discuss possible generalizations of the model toward the end of the section.

\section{$2.1 \quad$ Economy}

The economy is defined on a discrete time sequence, $t=0,1,2, \ldots$. There are two traded securities, a riskless bond and a stock. The bond is in unlimited supply at a constant, non-negative interest rate, $r$. The stock pays a dividend $D_{t+1}$ in period $t+1$, which consists of two additive components:

$$
D_{t+1}=F_{t}+G_{t} .
$$

Shares of the stock are traded in a competitive stock market. Let $P_{t}$ denote the exdividend price of the stock at time $t$.

There are two classes of investors, 1 and 2, with relative population weight of $\omega$ and $1-\omega$, respectively. Investors are identical within each class, but are different between the classes in their endowments and information. For convenience, an investor in class $i$ is referred to as investor $i$, where $i=1,2$.

Each investor is initially endowed with $\bar{x}$ shares of the stock. He is also endowed with a flow of income from a nontraded asset. In period $t$, investor $i$ has $Z_{t}^{(i)}$ units of the nontraded asset that pays $N_{t+1}$ per unit in the subsequent period.

At time $t$, all investors observe the current dividend of the stock $\left(D_{t}\right)$, its price $\left(P_{t}\right)$, the current payoff of the nontraded asset $\left(N_{t}\right)$, and their own endowment of the nontraded asset $\left(Z_{t}^{(i)}\right.$ for investor $\left.i\right)$. They also observe $F_{t}$, the forecastable part of each stock's next-period dividend. In addition, class 1 investors observe $G_{t}$. Thus, they have private information about future stock payoffs. The information set of investor $i$ at time $t$ is then given by $\mathcal{I}_{t}^{(1)}=\left\{D, P, N, F, G, Z^{(1)}\right\}_{[0, t]}$ and $\mathcal{I}_{t}^{(2)}=\left\{D, P, N, F, Z^{(2)}\right\}_{[0, t]}$, where $\{\cdot\}_{[0, t]}$ denotes the history of a set of variables from time 0 to $t$.

Each investor maximizes expected utility over his wealth next period of the following form:

$$
\mathrm{E}\left[-e^{-\lambda W_{t+1}} \mid \mathcal{I}_{t}^{(i)}\right]
$$

where $\lambda>0$ is the risk-aversion parameter.

All shocks (i.e., $\left\{F_{t}, G_{t}, N_{t}, Z_{t}^{(1)}, Z_{t}^{(2)} \forall t\right\}$ ) are assumed to be normally distributed with zero mean and constant variances: $\sigma_{F}^{2}$ for $F_{t}, \sigma_{G}^{2}$ for $G_{t}, \sigma_{N}^{2}$ for $N_{t}$, and $\sigma_{Z}^{(i) 2}$ for $Z_{t}^{(i)}$, respectively, where $i=1,2$. Furthermore, they are assumed to be mutually independent 
(contemporaneously and over time), except for $D_{t}$ and $N_{t}$, which are correlated with $\mathrm{E}\left[D_{t} N_{t}\right]=\sigma_{D N}$. In addition, for convenience in exposition, we set the riskless interest rate at zero and each investor's initial endowment of stock shares at zero $(\bar{x}=0)$. (Thus, the total supply of the stock is zero.) Without loss of generality, we set the investors' risk aversion $\lambda$ at one. ${ }^{3}$

The model defined above captures two important motives for trading: allocation of risk and speculation on future returns. Each investor holds the stock and the nontraded asset in his portfolio. Since the returns on the two assets are correlated, as his holding of the nontraded asset changes, each investor wants to adjust his stock positions to maintain an optimal risk profile. This generates allocational trade in the model, which we refer to as hedging trade. ${ }^{4}$ In addition, some investors might have private information about future stock payoffs. As new private information arrives, they take speculative positions in the stock in anticipation of high returns. This generates the informational trade in the model that we refer to as speculative trade.

\subsection{Equilibrium Price and Volume}

Given a stock price process $\left\{P_{t}\right\}$, the dollar return on one stock share is given by

$$
R_{t} \equiv D_{t}+P_{t}-P_{t-1} \quad(t=1,2, \ldots)
$$

The return consists of two parts, a dividend and a capital gain. Let $\mathrm{E}_{\mathrm{t}}^{(i)}\left[R_{t+1}\right]$ denote investor $i$ 's conditional expectation of $R_{t+1}$ given his information at $t, \sigma_{R}^{(i)} 2$ its conditional variance, and $X_{t}^{(i)}$ his stock holding. (Here, $\sigma_{R}^{(i)}$, the conditional variance of stock returns for investor $i$, has no time subscript because it remains constant over time.) We have the following proposition:

Proposition 1 The economy defined above has an equilibrium in which investor $i$ 's stock holding is

$$
X_{t}^{(i)}=\frac{\mathrm{E}_{\mathrm{t}}^{(i)}\left[R_{t+1}\right]}{\sigma_{R}^{(i)_{2}}}-\frac{\sigma_{D N}}{\sigma_{R}^{(i)_{2}}} Z_{t}^{(i)}=\frac{\mathrm{E}_{\mathrm{t}}^{(i)}\left[D_{t+1}\right]-P_{t}}{\sigma_{R}^{(i)_{2}}}-\frac{\sigma_{D N}}{\sigma_{R}^{(i)_{2}}} Z_{t}^{(i)} \quad(i=1,2)
$$

\footnotetext{
${ }^{3}$ Our model, which uses Constant Absolute Risk Aversion preferences and normally distributed shocks, exhibits homotheticity. That is, the implications of the model are invariant to proportional scaling of the variances of all the shocks and the investors' risk aversion. Thus, it is convenient to express the results to reflect this invariance. We choose to let $\lambda=1$ and thank the referee for suggesting this.

${ }^{4}$ Many papers have introduced nontraded assets to generate investors' hedging needs to trade in the market. See, for example, Bhattacharya and Spiegel (1991) and Wang (1994).
} 
and the ex-dividend stock price is

$$
P_{t}=F_{t}+\tilde{P}_{t} \equiv F_{t}+a G_{t}-\left(b^{(1)} Z_{t}^{(1)}+b^{(2)} Z_{t}^{(2)}\right)
$$

where $\mathrm{E}_{\mathrm{t}}^{(1)}\left[D_{t+1}\right]=G_{t}, \mathrm{E}_{\mathrm{t}}^{(2)}\left[D_{t+1}\right]=\gamma\left(\tilde{P}_{t}-b^{(2)} Z_{t}^{(2)}\right)$ and $a, b^{(1)}, b^{(2)}, \sigma_{R}^{(1) 2}, \sigma_{R}^{(2)} 2$, and $\gamma$ are constants.

Each investor's stock holding has two components. The first component is proportional to his risk tolerance and the risk-adjusted, expected stock return given his information. This component reflects the optimal trade-off between the return and risk of the stock. The second component is proportional to the amount of his non-traded asset, and reflects his need to hedge the non-traded risk.

The equilibrium stock price at time $t$ depends on $F_{t}$ and $G_{t}$, and on the amounts of both investors' non-traded asset, $Z_{t}^{(1)}$ and $Z_{t}^{(2)}$, respectively. $F_{t}$ gives the expected nextperiod dividend based on (non-price) public information. $G_{t}$ reflects class 1 investors'

private information on the next dividend. $Z_{t}^{(1)}$ and $Z_{t}^{(2)}$ give the investors' need to use the stock to hedge their nontraded risk.

An investor changes his stock position when there is a change in his expectation of future stock returns or his exposure to nontraded risk. This generates trading in the market. Given that trading is only between the two classes of investors, the volume of trade, $V_{t}$, is given by the change in the total stock holdings of either class. Thus,

$$
V_{t}=\omega\left|X_{t}^{(1)}-X_{t-1}^{(1)}\right|=(1-\omega)\left|X_{t}^{(2)}-X_{t-1}^{(2)}\right| .
$$

\subsection{Dynamic Relation Between Return and Volume}

In our model, returns are generated by three separate sources: public information on future payoffs, investors' hedging trades and their speculative trades. The returns generated by different sources exhibit different dynamics.

Returns generated by public news on future payoffs are independent over time. As public information about future dividends arrives (i.e., the realization of $F_{t}$ at $t$ ), the stock price changes to fully reflect the public information. As Section 2.2 shows, the price change has no impact on investors' stock demands, despite changes in their wealth. As a result, expected future returns remain unchanged. In other words, public news on future payoffs results in a white noise component in stock returns.

Returns generated by trading are serially correlated. When investors trade for hedging reasons, the stock price adjusts to attract other investors to take the other side. This 
price change contains no information about the stock's future payoffs. Thus, a price change generated by a hedging trade implies future returns of the opposite sign. For example, when class 1 investors sell the stock to hedge their nontraded risk, the stock price decreases, yielding a negative return for that period. However, the expected payoff in the next period stays the same. Hence, the decrease in the price leads to an increase in the expected return in the next period. Thus, returns generated by hedging trades tend to reverse themselves.

When investors trade for speculative reasons, the price changes to reflect the informed investors' expectation of the stocks' future payoffs. This expectation is fulfilled later on as private information becomes public. Thus, a price change generated by speculative trade implies future returns of the same sign. For example, when class 1 investors sell the stock due to a negative signal on future stock dividends, the stock price decreases, yielding a negative return for the current period. Since the price only partially reflects the private information (in a non-fully revealing equilibrium), the return in the next period is more likely to be negative as the private information becomes public. Thus, returns generated by speculative trade tend to continue themselves.

The actual dynamics of returns depend on the relative importance of the three returngenerating mechanisms. We are interested in returns generated by trading, with particular attention to the relative amount of hedging trade versus speculative trade and their relative impact on stock prices. By analyzing the serial correlation of returns generated by trading among investors, we could learn about the relative importance of different trading motives. Therefore, we would like to separately identify the returns generated by trading from those generated by public news on future payoffs, and examine their dynamics. We use trading volume to facilitate this identification. We observe that in our model, price changes generated by speculative or allocational trading must be accompanied by high volume, but those generated by public news about payoffs do not. In other words, by conditioning on the current volume return pair, we can (imperfectly) identify trade-generated returns. (See CGW for a discussion of this point.) Based on those returns, we can further examine how they might predict future returns. When all trades are hedging trades, current returns together with high volume predict strong reversals in future returns (as shown in CGW). When speculative trades are more important, current returns together with high volume predict weaker reversals (or even continuation) in future returns, as suggested in Wang (1994).

To analyze more formally how the relative importance of hedging trade versus spec- 
ulative trade might affect return dynamics, we first compute the expectation of future returns conditioned on both current return and volume. We present our result in the following proposition:

Proposition 2 From (5),

$$
\mathrm{E}\left[R_{t+1} \mid \tilde{V}_{t}, R_{t}\right]=\beta_{1} R_{t}-\beta_{2} \tilde{V}_{t} \tanh \left(\eta \tilde{V}_{t} R_{t}\right)
$$

where $\tilde{V}_{t} \equiv V_{t} / \mathrm{E}\left[V_{t}\right]$ is volume normalized by its unconditional mean and $\beta_{1}, \beta_{2}$, and $\eta$ are constants. Moreover, $\beta_{2} \geq 0$ and $\eta \geq 0$.

Equation (7) forecasts future by returns using current return and volume. It is obvious that given the current return, the higher the current trading volume implies stronger reversal in the next return. ${ }^{5}$ We can further consider an approximation of the forecasting equation when volume and return are small:

$$
\mathrm{E}\left[R_{t+1} \mid \tilde{V}_{t}, R_{t}\right]=\left(\theta_{1}-\theta_{2} \tilde{V}_{t}^{2}\right) R_{t}+\text { higher-order terms in } \tilde{V}_{t} \text { and } R_{t}
$$

where $\theta_{1}=\beta_{1}$ and $\theta_{2}=\beta_{2} \eta \geq 0$. Equation (8) clearly illustrates the dynamic relation between current return and volume and future return: Volume is related to return autocorrelations. Even though this result can be stated in the general form of Equation (7), we use the approximate form of Equation (8) in our future analysis for its intuitive appeal. Given the small magnitudes of average daily volume and returns of individual stocks, this approximation is reasonable.

Next, we examine how the dynamic volume-return relation in Equation (8) might depend on the importance of speculative trade in the market, which is driven by information asymmetry. For clarity of exposition, we set $Z_{t}^{(2)}=0$ for the rest of this section. Thus, class 1 investors generate all the trades.

A natural measure of information asymmetry is $\sigma_{G}^{2}$, the variance of the dividend component on which informed investors have private information. Thus, we consider how $\theta_{2}$ changes with $\sigma_{G}^{2}$, holding constant the total risk of the stock. The (unconditional) risk of the stock has two components: uncertainty in dividend, given by $\sigma_{D}^{2} \equiv \sigma_{F}^{2}+\sigma_{G}^{2}$, and uncertainty in future price, given by $\sigma_{\tilde{P}}^{2}$ for the uninformed investors.

When $\sigma_{G}^{2}=0$, there is no information asymmetry and investors trade only to hedge their nontraded risk. When $\sigma_{G}^{2}>0$, there is information asymmetry and the informed

\footnotetext{
${ }^{5}$ In the simple specification of the model, we set the total supply of the stock $(\bar{x})$ at zero. Thus, the unconditional mean of the excess stock return is zero. Since this paper focuses on the dynamics of stock returns, this simplification does not affect our results.
} 
investors trade for hedging and speculative reasons. We then have the following proposition:

Proposition 3 For $\sigma_{G}^{2}=0$,

$$
\theta_{2}=\theta_{20} \equiv \frac{\sigma_{\tilde{P}}^{2}}{\pi \sigma_{D}^{2}}=\frac{\omega^{2}}{\pi} \frac{\sigma_{D N}^{2} \sigma_{Z}^{2}}{\sigma_{D}^{2}}
$$

For $\sigma_{G}^{2}>0$ but small and holding $\sigma_{D}^{2}, \sigma_{\tilde{P}}^{2}$ constant,

$$
\theta_{2}=\theta_{20}\left[1-\omega\left(\frac{1}{\sigma_{D}^{2}}+\frac{3}{2 \sigma_{\tilde{P}}^{2}}\right)\right]+o\left(\sigma_{G}^{2}\right)
$$

which decreases with $\sigma_{G}^{2}$.

Thus, holding constant the risk of the stock, $\theta_{2}$ decreases with the degree of information asymmetry between investors, which is measured by $\sigma_{G}^{2}$.

Although this result is stated only for a small $\sigma_{G}^{2}$ when an analytical proof is available, we also examine its validity numerically when $\sigma_{G}^{2}$ is large. By computing $\theta_{2}$ for the complete range of $\sigma_{G}$ (between zero and $\sigma_{D}^{2}$ ) for a wide range of parameter values (of $\sigma_{D}^{2}$, $\sigma_{Z}^{2}$, and $\sigma_{D N}$ ), we find that the dependence of $\theta_{2}$ on $\sigma_{G}^{2}$ is always negative. ${ }^{6}$

Propositions 2 and 3 show how current return and volume predict future returns, and how this predictability depends on the relative significance of hedging trade versus speculative trade. In a cross-sectional context, all else equal, stocks with higher information asymmetry will have lower $\theta_{2}$ than will stocks with lower information asymmetry. It is this dependence of the return-volume dynamics on the degree of information asymmetry in the market, characterized in Equations (8) and (9), that we test empirically.

\subsection{Discussion of the Model}

Our model is similar to that of Wang (1994) with two simplifying assumptions. First, shocks to the economy are independently (and identically) distributed over time. The independence assumption implies that investors' private information is short-lived: It is only about the next dividend, which is revealed after one period. Thus, the less-informed investors do not have to solve the dynamic learning problem (and the corresponding optimization problem for their stock demand), which simplifies their policy. ${ }^{7}$ Second,

\footnotetext{
${ }^{6}$ Results of the numerical analysis are available upon request.

${ }^{7}$ For different cases of long-lived private information in a competitive setting, see, for example, Brown and Jennings (1989), Grundy and McNichols (1989), He and Wang (1995), and Wang (1994).
} 
we assume that investors are myopic, which further simplifies the investors' optimization problem.

Our simplification gives sharper results about the dependence of the volume-return relation on information asymmetry, as shown in Proposition 3, while Wang (1994) relies on numerical analysis and provides only examples. However, our model has the restrictive implication that $\theta_{2}$ remains non-negative even for high degrees of information asymmetry. As shown in Wang (1994), when private information can be long-lived, $\theta_{2}$ can become negative as the degree of information asymmetry increases. In our empirical analysis, we allow this possibility.

We assume that investors have constant absolute risk-aversion (CARA) and that assets (the stock and the nontraded asset) have (conditionally) normally distributed payoffs. The combination of these two assumptions allows closed form solutions for the model. However, as a special feature of the CARA preferences, each investor's stock demand is independent of his wealth. Hence, there is no income effect in investors' stock demand, and public news on future asset payoffs (and the corresponding price change) does not cause investors to trade in the market. Of course, for more general preferences, investors do rebalance their portfolios in response to public news on future payoffs as their wealth changes, giving rise to another motive for allocational trades. As mentioned earlier, our model introduces a motive for allocational trade by including a set of nontraded assets in investors' portfolio. We note that the detailed motive for allocational trades is not crucial to our main result. This particular choice in the model is for tractability and simplicity.

Despite the simplifying assumptions of the model, it provides a clear illustration of certain volume-return relations, which we believe to be more general than the model itself. As discussed above, relaxing many of these assumptions is possible, but adds little to the main thrust of the paper. ${ }^{8}$

\section{Empirical Methodology}

\subsection{Data and Sample Description}

Our primary sample comprises of all common stocks traded on the NYSE and AMEX. From CRSP, we obtain data for daily return, price, number of shares traded and shares

\footnotetext{
${ }^{8}$ There are assumptions in the model that are more substantial, such as those on the preferences and distribution combination and the information structure. Relaxing those would significantly change the model.
} 
outstanding. We obtain quotes and bid-ask spreads data from the TAQ database. Our sample period is from January 1st, 1993 to December 31st, 1998. We choose this six-year sample period for two reasons. First, the TAQ database only starts at the beginning of 1993. Second, the nature of our test requires that stock-specific parameters remain constant over time, which may not be the case over a long period. ${ }^{9}$ During the sample period, the CRSP database contains 3,538 stocks that were traded on the NYSE and AMEX. To allow a more precise estimation of our time-series regressions, and a more uniform cross-sectional comparison of the parameters, we further require that stocks in the sample trade in at least two thirds of the days (1,000 days out of 1,516 possible trading days). This requirement reduces our sample to 2,226 stocks.

Table 1 presents firms' characteristics for the entire sample and for three subgroups according to size. For each firm $i$, we measure size $\left(\operatorname{AvgCap}_{i}\right)$ as the average daily market capitalization (number of shares outstanding multiplied by the daily closing price) over the sample period. The market capitalization of firms in our sample ranges from $\$ 3.61 \mathrm{M}$ to $\$ 147.82 \mathrm{~B}$. As indicated by the columns 2 and 3 of the table, both the average daily number of shares traded $\left(\operatorname{Avg} \operatorname{Trd}_{i}\right)$ and the average share turnover $\left(\operatorname{AvgTurn}{ }_{i}\right)$, which is the number of shares traded relative to shares outstanding, increase with firm size. For example, the daily average turnover is $0.27 \%$ for the Small size group and is $0.355 \%$ for the Large size group. The average share price $\left(\mathrm{AvgPrc}_{i}\right.$ in column 4$)$ exhibits the same pattern.

Using the data from TAQ, we construct a measure of a stock's bid-ask spread (BAsprd $\left.{ }_{i}\right)$. In light of results in Madhavan, Richardson, and Roomans (1997), we use the opening spread to capture the asymmetric information component of the spread. We then define the relative spread for each stock as the average of the daily opening percentage spread (opening bid-ask spread divided by the opening mid-quote) over the sample period. Consistent with many prior studies, the relative spread is high for the Small size group $(4.11 \%)$, and decreases monotonically with firm size (the average for firms in the Large size group is only $0.84 \%$ ).

\subsection{Return, Volume, and Proxies for Information Asymmetry}

We use daily returns and trading volume to analyze the impact of information asymmetry on the dynamic volume-return relation. The main reason to use daily data is to be able to

\footnotetext{
${ }^{9}$ In Section 4.2, we show that increasing the time horizon to ten instead of six years does not affect our results.
} 
relate our results to those of previous studies (e.g., Antoniewicz, 1993; CGW; LeBaron, 1992; and Stickel and Verrecchia, 1994). ${ }^{10}$ In Section 4.2 we consider an alternative procedure to determine the appropriate time interval for our analysis empirically. The return series we use for the estimation is the daily return of individual stocks from CRSP (we test the sensitivity of our results to an alternative definition of returns that avoids the potential bid-ask bounce problem in Section 4.3).

We use daily turnover as a measure of trading volume for individual stocks. We define a stock's daily turnover as the total number of shares traded in that day divided by the total number of shares outstanding. ${ }^{11}$ Since the daily time series of turnover is nonstationary, we measure turnover in logs and detrend the resulting series. To avoid the problem of zero daily trading volume, we add a small constant (0.00000255) to the turnover before taking logs. ${ }^{12}$ We detrend the resulting series by subtracting a 200-trading-day moving average:

$$
V_{t}=\log _{\text {turnover }}-\frac{1}{200} \sum_{s=-200}^{-1} \text { logturnover }_{t+s}
$$

where

$$
\operatorname{logturnover}_{t}=\log \left(\text { turnover }_{t}+0.00000255\right) \text {. }
$$

Panel B of Table 1 provides summary statistics that describe the volume series used in the estimation. While the first-order autocorrelation is higher for larger stocks, the pattern becomes less pronounced in the fifth order and disappears in the tenth order. We test the sensitivity of our results to alternative definitions of volume in Section 4.4.

As in CGW, we note that there is some slippage between the theoretical variables in the model and those in the empirical part. Our model considers dollar returns per share and normalized turnover, and the empirical analysis considers returns per dollar and detrended log turnover for well-known reasons (e.g., potentially better distributional properties). The difference between the theoretical and corresponding empirical variables

\footnotetext{
${ }^{10} \mathrm{CGW}$ also consider the same relation between two-day returns and volume for the market and find it is still present, but weaker than that for daily data. However, the analysis of Conrad, Hameed, and Niden (1992) and Lee and Swaminathan (2000) indicate that volume can be informative for returns over longer horizons.

${ }^{11}$ Lo and Wang (2000) provide a theoretical justification for using turnover as a measure of trading volume and a detailed study of the turnover of individual stocks.

${ }^{12}$ The value of the constant is chosen to maximize the normality of the distribution of daily trading volume. See Richardson, Sefcik, and Thompson (1986), Cready and Ramanan (1991), and Ajinkya and Jain (1989) for an explanation.
} 
is mainly a matter of normalization. At the daily frequency that we focus on, the relation among these variables should not be very sensitive to the normalizations used here.

To test Proposition 3, we also need a measure of information asymmetry for individual stocks that represents the extent of informed trading. Since information asymmetry is not directly observable, we must find a suitable proxy for the empirical investigation. Previous studies use several variables to measure information asymmetry, among which are bid-ask spreads and market capitalization. Some researchers argue that firms with lower bid-ask spreads (e.g., Lee, Mucklow, and Ready, 1993) and larger firms (e.g., Lo and MacKinlay, 1990) have a lower degree of information asymmetry or smaller adverse selection costs. We use both proxies in our empirical analysis for a couple of reasons. First, since there is no agreement on which proxy is the "best," we believe it is prudent not to rely on only one of them. Second, by using more than one proxy, we can examine the sensitivity of our results to various empirical representations of information asymmetry. For this reason, we employ in Section 4.5 yet another proxy, the number of analysts who are following a stock, which is linked to the degree of information production in the market. Recent studies (Brennan and Subrahmanyam, 1995; and Easley, O'Hara, and Paperman, 1998) find that firms with a larger analyst following have a lower degree of information asymmetry or lower adverse selection costs. Hence, we use the number of analysts as a proxy for information asymmetry, and we expect that the more analysts who are following a stock, the less information asymmetry there is about it.

Even with an agreement on the proxies, there is still the question of how to use these proxies in a cross-sectional test. The exact nature of the functional relation between information asymmetry and the proxy is unknown. For example, the last column in Panel A of Table 1 indicates that while the average bid-ask spread of the Large size group is five times smaller than that of the Small size group, the average firm size of the Small group is almost 100 times smaller than that of the Large group (first column). To work with the two proxies in a unified framework, we adopt an ordinal transformation of the variables. That is, we order the firms in an ascending order according to the proxy and assign a rank of one to the first firm (say, the smallest firm when firm size is the proxy), and a rank of 2,226 to the last firm. We then divide the ordinal variable by 2,226 so that its range is between zero and one. This monotonic transformation preserves the intuition of the differences between low and high information asymmetry without reading too much into the specific differences in magnitude. ${ }^{13}$

\footnotetext{
${ }^{13}$ See Johnston (1985) and references therein for a justification of this transformation.
} 
The correlation between ORDCAP and ORDBA (the variables representing the ordinal scales of AvgCap and BAsprd) is -0.876. Although this is a moderately high correlation, it does not suggest that the two proxies represent the exact same phenomenon. We have also repeated our experiments using either the raw variables (AvgCap and BAsprd) or their log transformations. Our results are not materially affected by these alternative representations.

\subsection{Experiment Design}

To test Proposition 3, we estimate the following relation for each individual stock:

$$
R_{i t+1}=\mathrm{C}_{i}+\mathrm{C} 1_{i} \cdot R_{i t}+\mathrm{C} 2_{i} \cdot R_{i t} V_{i t}+\text { error }_{i t+1}
$$

While the relation in Equation (8) has squared normalized volume entering the interaction term, we estimate the relation with our definition of normalized volume, the detrended log turnover, without squaring. We do this to allow for comparisons with prior empirical studies (e.g., CGW). We test the relation in Equation (8) with a measure of squared volume in Section 4.4 .

In principle, trading in every stock contains both hedging and speculative elements. The observed volume-return relation depends on the relative importance of one type of trade relative to the other. We should see statistically significant positive $\mathrm{C} 2$ coefficients for stocks that are associated with very significant speculative trade, while for stocks with predominantly hedging trade, the $\mathrm{C} 2$ coefficients should be clearly negative. Stocks for which neither speculative nor hedging trade dominates should have $\mathrm{C} 2$ coefficients that are insignificantly different from zero. Moreover, the relation between $\mathrm{C} 2$ and the significance of speculative trade relative to hedging trade is monotonic.

To examine the relation between the importance of information asymmetry and the C2 coefficients, we use both structured and nonstructured methods. To give a sense of the underlying relation without imposing additional structure, we present a discrete categorization analysis of the results by assigning the stocks into three groups of the information asymmetry proxy. Proposition 3 implies the following relation:

$$
\mathrm{C} 2_{i}=f\left(\mathrm{~A}_{i}\right)
$$

where $A_{i}$ is a proxy for the degree of information asymmetry of an individual stock. For the bid-ask spread proxy, higher values of $\mathrm{A}_{i}$ are associated with a higher degree of information asymmetry, and we should observe that the mean of $\mathrm{C} 2{ }_{i}$ is more positive for 
stocks with larger bid-ask spreads. For the market capitalization proxy, higher values of $\mathrm{A}_{i}$ are associated with a lower degree of information asymmetry, and so the mean of $\mathrm{C} 2{ }_{i}$ should be more positive for smaller stocks.

Under the assumption that the relation is linear, we can estimate the cross-sectional relation

$$
\mathrm{C} 2{ }_{i}=a+b \cdot \mathrm{A}_{i}+\text { error }_{i}
$$

Here, we should see $b>0$ when the information proxy used is the bid-ask spread and $b<0$ when the information proxy used is market capitalization.

\section{Empirical Results}

\subsection{Tests of the Dynamic Volume-Return Relation}

Table 2 presents the results of using the bid-ask spread as the information asymmetry proxy. For each stock in the sample, we estimate the parameters $\mathrm{C} 0, \mathrm{C} 1$, and $\mathrm{C} 2$ of Equation (10). In Panel A, we present summary statistics for these 2226 time-series regressions for each of the three bid-ask spread groups. The parameter that interest us is the coefficient on the interaction term between return and volume, C2. As Proposition 3 predicts, the mean value of $\mathrm{C} 2$ decreases monotonically as we go from stocks with large bid-ask spreads to stocks with small bid-ask spreads. Stocks with a higher information asymmetry (large bid-ask spreads) are associated with positive coefficients (0.035 for the High group). The mean value becomes negative for stocks in the Low group (-0.003). The nonparametric analysis points in the same direction: Only 141 (out of 742) of the stocks in the High group have negative coefficients, compared with 378 in the Low group.

As we expected, most C2 coefficients of firms with large bid-ask spreads are positive and statistically different from zero, indicating the importance of speculative trade. For many of the stocks with medium spreads, the C2 coefficients are not significantly different from zero, which is consistent with a balance of both speculative and hedging trades. For stocks with small bid-ask spreads, many C2 coefficients are negative and statistically significant, indicating the dominance of hedging trades. The evidence in the table points to a monotone positive relation between $\mathrm{C} 2$ and bid-ask spreads.

In Panel B, we use regression analysis to examine this relation. Equation (12) is estimated using the bid-ask spread as the information asymmetry proxy, i.e., the dependent variable is $\mathrm{C} 2$ (the influence of volume on the autocorrelation of returns) and the 
independent variable is ORDBA (the bid-ask spread rank order). The spread coefficient is positive and highly significant, indicating that stocks with small spreads (i.e., lower information asymmetry) have lower volume-return interaction terms. We note that when ORDBA is used as the information asymmetry proxy, we can explain over $10 \%$ of the cross-sectional variation in $\mathrm{C} 2$.

In Table 3, we use size (market capitalization) to proxy for information asymmetry. The results are similar to those in Table 2. Because larger size is associated with lower information asymmetry, the interaction coefficient $\mathrm{C} 2$ is the most positive for small firms (high information asymmetry) and decreases as the size of the firm increases. In the Low group, 167 stocks have negative $\mathrm{C} 2$ coefficient (mean value 0.030 ), but there are 354 stocks with negative $\mathrm{C} 2$ coefficient in the High group with a mean that is very close to zero. The regression results in Panel B tell the same story: There is a statistically significant negative relation between our proxy for information asymmetry and the volume-return interaction parameter.

The results in these two tables are consistent with the prediction of Proposition 3. Using two different information proxies, we find that following high volume, stocks that are associated with more informed trading exhibit persistence in their returns and stocks with less informed trading exhibit reversals.

While we attribute the cross-sectional variation in C2 to different degrees of information asymmetry (or speculative trading), it may also be attributed to other factors such as differences in liquidity across stocks. In particular, for less-liquid stocks, high volume is associated with a higher price impact and a larger subsequent return reversal than for more-liquid stocks. Hence, less-liquid stocks should have more negative C2 coefficients. However, natural candidates for stocks with lower liquidity are those stocks with small market capitalizations or large bid-ask spreads. Hence, liquidity considerations should cause a larger return reversal following high-volume days for lower market capitalization or large bid-ask spread stocks. This is the opposite of what we find. If a liquidity effect exists, our empirical findings suggest that it is dominated by the information effect.

For the results presented in Tables 2 and 3, we estimate both the time-series and the cross-sectional relations by using OLS. One possible concern is whether this experiment design is robust to potential econometric problems. One econometric problem could be that the estimated relation in the time-series regression is affected by autocorrelated errors. In this case, a lagged dependent variable among the regressors precludes using OLS for the estimation. To examine how this problem might affect our results, we use a test 
developed by Breusch (1978) and Godfrey (1978a, 1978b) to identify the most appropriate error structure. For each stock, we test for white noise against the alternative of an autoregressive error structure of orders one through five. We decided to limit the possible orders to five after a lengthy inspection of some stocks. We use a $5 \%$ significance level to reject the white noise hypothesis. If the test is significant for any order $p<5$, but not for higher orders, we test again with the null of $\operatorname{AR}(p)$ against an autoregressive structure of orders higher than $p$, but only up to order five. After identifying the appropriate order, we estimate the relation in Equation (10) using maximum likelihood with the suitable autoregressive structure. We perform this procedure separately for each stock.

Using these time-series estimated C2 coefficients, we then re-run the cross-sectional regressions with the information asymmetries proxies as the dependent variables. The results are presented in Table 4, Panel A. These results are similar to the OLS findings reported in Tables 2 and 3, and show the same strong relation with the information asymmetry proxies. Therefore, our results do not appear to be are sensitive to autocorrelation of the error terms in the time-series estimations. To assess the sensitivity of our results to the order identification algorithm, we repeat all estimations identifying the appropriate error structure only by the white noise test against an AR structure. Panel B of Table 4 presents the results of this specification. Our results appear robust to the exact manner in which the appropriate autoregressive order is identified.

Another possible econometric problem is that if the errors of Equation (10) are correlated across stocks, the $\mathrm{C} 2{ }_{i}$ estimates will not be independent. When we estimate Equation (12), the standard error of $b$ is then biased and tests of significance are difficult to interpret. Because a cross-correlation of the errors most likely arises from the sensitivity of the returns to missing common factors, one way to decrease such cross-correlation is to model the factors directly. Following Jorion (1990), we use a market proxy to model the missing common factors for the purpose of decreasing cross-correlations of the error terms. We estimate the following time-series relation for each stock:

$$
R_{i t+1}=\mathrm{D} 0_{i}+\mathrm{D} 1_{i} \cdot R_{i t}+\mathrm{D} 2_{i} \cdot R_{i t} V_{i t}+\mathrm{D} 3_{i} \cdot R_{m t+1}+\operatorname{error}_{i t+1}
$$

where $R_{m t+1}$ is the return on a value-weighted portfolio comprised of all common stocks that trade on the NYSE or AMEX, and which have valid return and volume information in the CRSP database for that day. We then estimate the cross-sectional relation:

$$
\mathrm{D} 2_{i}=a+b \cdot \mathrm{A}_{i}+\text { error }_{i}
$$


Table 5 reports the results of estimating Equation (14). The $b$ coefficient that measures the relation between the information asymmetry proxy and the influence of volume on return autocorrelations is positive and significant for ORDBA, and negative and significant for ORDCAP.

Another potential problem has its root in Equation (8) from Proposition 2 where the dynamic volume-return relation is developed using an approximation that ignores higher-order, nonlinear terms in the product of volume and return. if the product is very large, the approximation may not be good. We could trim observations above a certain bound, but the problem is to choose a sensible bound. So the approach we choose to take is to use an econometric methodology that identifies observations that are too large relative to a linear structure (i.e., outliers that could be the results of nonlinearities) and eliminate them from the analysis. The methodology that we use is the two-stage least trimmed squares (see Rousseeuw and Leroy, 1987). In the first stage, the least trimmed squares (LTS) estimator is applied to the relation. The estimator minimizes the sum of the smallest $h$ residuals, where $h=\frac{2}{3} n$ and $n$ is the number of observations. We use the residuals from the LTS estimation to create weights that identify an observation as an outlier if its residual is too large relative to a measure of the standard errors. ${ }^{14}$ In the second stage, we use these weights in a weighted least squares (WLS) estimation of the cross-sectional relation. We then report the results that come out of the second stage estimation.

The two-stage LTS therefore enables us to estimate a cleaner linear relation. It is much less influenced by possible nonlinearities that might produce observations that are too far from the linear approximation, and which could result in biased slope coefficients. Table 6 presents the results of applying the above procedure to our data. In Panel A, we estimate each time-series regression using the two-stage LTS methodology. Then,

${ }^{14}$ The LTS estimator is given by:

$$
\min _{\hat{\theta}} \sum_{i=1}^{h}\left(r^{2}\right)_{i: n}
$$

where $\hat{\theta}$ is the vector of estimated parameters and $\left(r^{2}\right)_{1: n} \leq \cdot \leq\left(r^{2}\right)_{n: n}$ are the ordered squared residuals. The weights are then defined as:

$$
w_{i}=\left\{\begin{array}{ll}
1 & \text { if }\left|r_{i} / \hat{\sigma}\right| \leq 2.5 \\
0 & \text { if }\left|r_{i} / \hat{\sigma}\right|>2.5
\end{array} \quad \hat{\sigma}=C_{2} \sqrt{\frac{1}{n} \sum_{i=1}^{h}\left(r^{2}\right)_{i: n}}\right.
$$

where $C_{2}$ is a correction factor. 
we estimate Equation (12), the cross-sectional regression, by using OLS. The results are similar to those reported in Tables 2 and 3: The coefficients of the information asymmetry proxies are highly significant and in the right direction. Hence, it does not seem as if very large observations are adversely affecting the estimates of the parameters C2.

Because we do not know the functional form of the relation between information asymmetry and the proxy we use, we can also apply the two-stage LTS approach to our cross-sectional estimation. We use the $\mathrm{C} 2$ estimates that come out of the OLS time-series estimation to allow for a cleaner comparison with the results in Tables 2 and 3. Panel B of Table 6 presents the result of estimating the cross-sectional regression using two-stage LTS. The results are similar to those of the OLS estimation.

Lastly, we repeat our cross-sectional analysis using the non-parametric Spearman correlations. We wish to measure the association between the $\mathrm{C} 2$ coefficients and the information asymmetry proxies without imposing the OLS assumptions (e.g., normality of the residuals). The Spearman correlation between C2 and ORDBA is 0.326 (asymptotic standard error 0.02), and the correlation between C2 and ORDCAP is -0.26 (asymptotic standard error 0.02). Hence, the Spearman correlations point to the same conclusions as all our other econometric procedures.

We can ask several questions about the results presented so far. First, how do our choices for the length of the time-series estimation period or the daily intervals for return and volume affect our results? Second, can our results be attributed to the effect of bidask bounce? Third, how sensitive are our results to the exact definition of volume that we use? Fourth, can we relate our findings about information asymmetry to a variable that is more directly associated with information production? Fifth, is firm-specific information asymmetry a driving force behind the dynamic volume-return relation or does the relation disappear when we eliminate market-wide variations? The following sections address these questions.

\subsection{Alternative Lengths of Time Intervals}

It is possible that the appropriate measurement period differs across stocks. For example, for an infrequently traded stock, the period could be several days, so that more trades are captured within the period. We might choose the appropriate measurement periods to equate the quantities of noise trading (on average) across stocks. The measurement interval is longer for stocks with little noise trading than it is for stocks with a lot of noise trading. While we do not have a measure of noise trading, we can use the typical 
turnover of a stock as a proxy for the normal level of trading. A typical trading intensity measured by the stock's median turnover is less sensitive to informational or allocational volume shocks.

Therefore, we calculate the median daily turnover for each stock over the sample period (MedTurn), and assign all stocks into three groups according to their median turnover. The average MedTurn for the three groups are $0.0634 \%, 0.1691 \%$, and $0.384 \%$, respectively. we note that the average MedTurn of the High group is about twice that of the Medium group and about five times that of the Low group. (The proportions are similar when we use the cross-sectional median rather the average of each group.) Therefore, given a daily interval for the most active stocks, we choose a two-day interval as the most appropriate for the Medium group, and a five-day interval for the Low turnover group. To calculate the return and turnover series for the Medium and Low groups, we compound returns and sum the turnover for the days in the interval.

We then perform a separate time-series analysis for each stock. A stock in the High MedTurn group that is listed for the entire sample period will have 1,516 observations in the regressions, while a similar stock in the Medium (Low) MedTurn group will have 758 (303) observations. Taking the C2 coefficient from each individual stock's time-series regression, we estimate the cross-sectional relation in Equation (12). We present the cross-sectional results in Table 7, Panel A. The bid-ask spread coefficient is positive and highly significant, and the size coefficient is negative and highly significant. Our results do not appear to be driven by the choice of the daily interval for the time-series regressions. Thus, whether we fix a time interval (a day in our experiment) or a given quantity of noise trading (as the current test implies) does not affect our findings.

In Panel B of Table 7, we use ten instead of six years to estimate the time-series regressions. This experiment allows us to check the sensitivity of our results to the length of the estimation period. We estimate the volume-return interaction parameters $(\mathrm{C} 2)$ in this panel by using data from 1989 through 1998. The coefficients of the information asymmetry proxies in the cross-sectional analysis have the right signs and are highly statistically significant. ${ }^{15}$

\footnotetext{
${ }^{15}$ While not reported in a table, we have also conducted the experiment with a sample of NYSE and AMEX stocks using the period 1983-1992. The results are the same as those presented in the paper.
} 


\subsection{Information Effects vs. Bid-Ask Bounce Effects}

Many studies show that short-horizon returns of individual stock exhibit negative autocorrelation (e.g., French and Roll, 1986; Lo and MacKinlay, 1988; Conrad, Kaul, and Nimalendran, 1991; Jegadeesh and Titman, 1995; Canina, Michaely, Thaler, and Womack, 1998). These autocorrelations are more pronounced in small stocks than in large stocks. French and Roll (1986) and Jegadeesh and Titman (1995) show that the first order autocorrelation of daily returns is negative for small stocks, increases with the size of the firm, and is positive for large firms. Our sample shows the same results. The firstorder autocorrelation of daily returns is negative for stocks with large bid-ask spreads $(-0.088)$ and small stocks (-0.076). It is positive but very small for large stocks (0.003) and stocks with small bid-ask spreads (0.01). These autocorrelations are similar in sign and relative magnitude to the $\mathrm{C} 1$ coefficients from Tables 2 and 3.

Lo and MacKinlay (1988) suggest that these empirical findings are consistent with security returns that reflect three influences: a positively autocorrelated common component, a white noise component, and a negative autocorrelation effect induced by microstructure phenomena such as bid-ask bounce. French and Roll (1986) suggest that the positive autocorrelation arises when the market does not incorporate information as soon as it is released. Jegadeesh and Titman (1995) attribute the negative autocorrelation to inventory control by specialists.

Our model shows how volume should interact with the autocorrelation of returns. For stocks with more information asymmetry, greater volume should make the first-order autocorrelation more positive (hence, a positive C2) due to the partial adjustment of prices to information. For stocks with less information asymmetry, greater volume should make the first-order autocorrelation more negative (hence a negative $\mathrm{C} 2$ ) due to the return reversal associated with liquidity shocks. However, for small stocks or stocks with large bid-ask spreads, the prediction of our model and the bid-ask bounce effect both operate in the same direction. We show that for stocks with large bid-ask spreads that exhibit negative return autocorrelation, increased volume makes the autocorrelation less negative (a positive $\mathrm{C} 2$ ). We claim that the positive $\mathrm{C} 2$ is the result of a high degree of information asymmetry. However, we would expect a positive C2 if more volume decreases the bid-ask bounce effect.

To examine this issue, we generate a return series that is free from bid-ask bounce. Using the TAQ database, we identify the end-of-day quote for all days in our sample period for all stocks (except Berkshire Hathaway Inc. that is excluded due to its abnormal price 
range). We then construct a return series from the mid-quotes, and adjust for stock and cash distributions using information from the CRSP database. We note that this return series is less reliable than the CRSP series used in the main analysis. First, there are more days without a valid end-of-day quote in the TAQ database than there are days without a valid return in the CRSP database. Each day without an end-of-day quote results in two days without valid mid-quote returns. Second, the intraday quote data in TAQ could contain more errors than the heavily used CRSP return series.

The mid-quote return series eliminates most of the negative autocorrelation in the returns of small stocks. For example, the first-order autocorrelation for the group of small stocks (742 firms) goes from -0.076 to -0.015 , and that of the group of stocks with large bid-ask spreads goes from -0.088 to -0.014 . Our goal is to estimate the timeseries regressions using the mid-quote returns, and then examine the impact of these new estimates on the cross-sectional relation that our model predicts (the relation between $\mathrm{C} 2$ and the information asymmetry proxies). We re-estimate Equation (10) with the midquote return series for the firms in our sample. An indication that the aforementioned problems with respect to the mid-quote return series might have some effect is that the time-series regressions using TAQ returns produce a few outliers of the $\mathrm{C} 2$ coefficient while the time-series regressions using CRSP returns do not produce any outliers. Hence, we estimate the cross-sectional relations using the two-stage LTS procedure described in Section 4.1 to identify and eliminate the influence of outliers. Table 8 presents the crosssectional regressions of ORDBA and ORDCAP on the interaction parameter $\mathrm{C} 2$. The information asymmetry proxies have the appropriate signs and are statistically significant. We note, though, that the proxies explain less of the variation in the $\mathrm{C} 2$ coefficients than they do in the cross-sectional regressions reported in Tables 2 and 3.

\subsection{Alternative Definitions of Volume}

Since there is a slight difference between our de-trended volume measure and the theoretical volume measure, Table 9 presents the results using alternative definitions of volume. In Panel $\mathrm{A}$, we define volume as the daily share turnover of a stock, without taking any transformation or detrending. We re-estimate the time-series relation in Equation (10) with this alternative volume definition. The results of the cross-sectional regressions show a statistically significant relation, in the appropriate direction, between $\mathrm{C} 2$ and both information asymmetry proxies.

In Panel B, we perform a more direct test of the relation in Equation (8) that comes 
out of our theoretical model. In the theoretical model, it is the squared volume, rather than a linear term, that affects the subsequent period's returns. We define volume as the logarithm of $(1+$ daily number of shares traded $) .{ }^{16}$ Then we estimate the following relation:

$$
R_{i t+1}=\mathrm{C}_{i}+\mathrm{C} 1_{i} \cdot R_{i t}+\mathrm{C} 2_{i} \cdot V_{i t}^{2} R_{i t}+\text { error }_{i t+1}
$$

The resulting cross-sectional analysis shows the same pattern as in Tables 2 and 3. In fact, it appears that the relation is even stronger. Both information asymmetry proxies explain over $16 \%$ of the cross-sectional variation in the parameter $\mathrm{C} 2$.

\subsection{Analyst Following as a Proxy for Information Production}

While the information asymmetry proxies we use in the main analysis have received much attention in the literature, several recent papers discuss the relation between the number of analysts who follow a stock and information asymmetry or adverse selection costs. Early papers used the number of analysts as a direct proxy for informed trading, but recent studies by Brennan and Subrahmanyam (1995), and Easley, O'Hara, and Paperman (1998) find that firms that are followed by a larger number of analysts have a lower degree of information asymmetry or lower adverse selection costs. ${ }^{17}$ Thus, the number of analysts appears to be negatively related to the degree of information asymmetry.

Using the number of analysts as a proxy for the degree of information asymmetry has an intuitive appeal since it directly relates to information production in the market. Nonetheless, this empirical proxy has its share of problems. First, there is still some doubt about the direction and strength of the proxy's relation to the degree of information asymmetry. Second, many stocks are not regularly followed by analysts. Third, the number of analysts is heavily influenced by membership in a particular industry. Fourth, there is relatively little cross-sectional variation in the number of analysts who follow stocks. Therefore, there are reasons to believe that the number of analysts will not exhibit as strong a cross-sectional relation with $\mathrm{C} 2$ as will our two main proxies, bid-ask spread and market capitalization.

To construct the analyst-following proxy, we look for the monthly number of analysts who provide I/B/E/S with end-of-fiscal-year earnings forecast for the current year. We

\footnotetext{
${ }^{16}$ To avoid taking the log of zero on days without trading, we add the small constant (1.00) to the daily number of shares traded before making the logarithm transformation.

${ }^{17}$ See Easley, O'Hara, and Paperman (1998) for a discussion of the issue and additional references.
} 
define NumEst ${ }_{i}$ to be the average monthly number of analysts over the sample period (six years). ORDEST ${ }_{i}$ is the ordinal scale of NumEst $_{i}$ (constructed like ORDBA and ORDCAP), where two firms that have the same number of analysts receive the same rank. Out of the 2,226 firms in our sample, 2,035 are followed by at least one analyst. Not surprisingly, the majority of firms without analyst coverage are in the Small size group. Only 571 out of 742 firms in the Small size group had forecast records in the $\mathrm{I} / \mathrm{B} / \mathrm{E} / \mathrm{S}$ database. The average number of analysts is 2.45 for those small firms that are being followed, compared with 16.51 for firms in the Large size group.

Table 10 contains the results of the cross-sectional regression in Equation (12) using either NumEst or ORDEST as the information asymmetry proxy. The coefficient of NumEst is negative and statistically significant and so is the coefficient of ORDEST, though the relation is weaker than the cross-sectional results reported in Tables 2 and 3 , where we use our two main proxies. Interpreting this result is straightforward. The more analysts who cover a firm, the better the production of information about the firm's prospects. Investors in a firm with more information production have fewer opportunities to engage in speculative trading, and therefore most trading in these firms' securities is motivated by hedging. Our results are consistent with the evidence in Brennan and Subrahmanyam (1995) and Easley, O'Hara, and Paperman (1998), who find that the number of analysts is negatively related to the degree of information asymmetry.

\subsection{Firm-Specific Information Asymmetry}

It is possible, and even likely, that both market-wide and firm-specific factors drive the trading and returns of individual stocks. In the model presented in Section 2, trading is only generated by the firm-specific hedging needs and private information. Thus, we focus on the different degree of firm-specific private information as the main factor that produces the cross-sectional variation in the dynamic volume-return relation. The model's prediction on the relation between volume and return autocorrelation would therefore most reasonably apply to the firm-specific components of trading and returns.

We can test empirically whether the volume-return relation has a firm-specific component or if it disappears when we eliminate market-wide variations. To do so, we use market models to decompose both the volume and return series. Each series is decomposed into a systematic (market) component and a non-systematic (firm-specific) component. To im-

plement the market models, we construct market return and volume series. The market's return for a specific day is defined as the return on a value-weighted portfolio comprised 
of all common stocks that traded on the NYSE or AMEX, and which have valid return and volume information in the CRSP database for that day. We define the market's turnover in an analogous fashion as the value-weighted average of the turnover of the individual stocks in the portfolio of all NYSE and AMEX common stocks. To maintain compatibility, we detrend the log market turnover series, just as we do with the turnover series of each individual stock. While sensible, this paper does not explicitly model this particular volume decomposition. In a recent study, Lo and Wang (2000) present a formal justification for a market model of volume, which we use here.

We re-estimate Equation (10) by using residual returns and residual volume from the respective market models. We then examine cross-sectional differences in the resulting $\mathrm{C} 2$ coefficients of the volume-return interaction terms. Table 11 presents the cross-sectional regressions of the information asymmetry proxies on the $\mathrm{C} 2$ coefficients. The coefficient of ORDBA is positive and statistically significant, and the coefficient of ORDCAP is negative and statistically significant. These results suggest that firm-specific information asymmetry is a driving force behind the relation between volume and return autocorrelations.

\section{Conclusions}

We construct a simple model in which investors trade in the stock market for both hedging and speculation motives. We use the model's insights to investigate the dynamic relation between volume and returns. According to our model, returns generated by hedgingmotivated trades reverse themselves, while returns generated by speculation-motivated trades tend to continue themselves. The relative significance of these two types of trades for an individual stock determines whether returns that are accompanied by trading volume exhibit negative or positive autocorrelation.

We test the model's predictions by using daily return and volume data for NYSE and AMEX stocks. We look at how volume affects the first-order autocorrelation of daily stock returns. To proxy for information asymmetry, we use bid-ask spreads (larger bidask spreads imply a higher degree of information asymmetry) and market capitalization (larger firms are associated with less information asymmetry).

The empirical results support the predictions of the model on the nature of the dynamic volume-return relation. Stocks that are associated with a high degree of informed trading exhibit more return continuation on high-volume days, and stocks that are associated with a low degree of informed trading show more return reversals on high-volume days. Our 
results are robust to various econometric specifications, alternative definitions of volume, and changes in the lengths of the measurement intervals and the estimation period. We also show that the dynamic volume-return relation remains significant after accounting for possible bid-ask bounce effects.

We use analyst following as an additional proxy for the degree of information production about a firm. We find that in the portion of our sample for which we could find data on analyst following, the dynamic volume-return relation shows the same pattern as with the other information asymmetry proxies. We also investigate whether firm-specific information asymmetry is a driving force behind this relation. We use market models to decompose returns and volume, and find that the relation holds even when we use only firm-specific (residual) returns and volume.

The empirical findings support the general notion that volume does tell us something about future price movements. The analysis also suggests that the actual dynamic relation between volume and returns depends on the underlying forces driving trading. Explicitly modeling these driving forces allows us to use volume effectively in making an inference about returns. In particular, by considering both allocational and informational trading, our model gives rise to realistic predictions that seem to encompass the variety that prevails in the market. It is this feature of the model that enables us to reconcile the previous empirical findings of return reversals after high-volume days exhibited by large firms and indices, with the return continuation after high-volume days shown by average firms. The key to generating both results is our ability to use information asymmetry to capture the cross-sectional variation in the dynamic volume-return relation of individual stocks. 


\section{Appendix}

Proof of Proposition 1. We consider the special case when $Z_{t}^{(2)}=0 \forall t$. Extending the result to the general case is straightforward. We re-express the price as:

$$
P_{t}=F_{t}+\tilde{P}_{t}
$$

where $\tilde{P}_{t} \equiv a\left(G_{t}-b Z_{t}\right), b \equiv b^{(1)} / a$ and $Z_{t} \equiv Z_{t}^{(1)}$. We have

$$
\mathrm{E}_{\mathrm{t}}^{(i)}\left[R_{t+1}\right]=\mathrm{E}_{\mathrm{t}}^{(i)}\left[D_{t+1}\right]-P_{t}=\mathrm{E}_{\mathrm{t}}^{(i)}\left[G_{t}\right]-\tilde{P}_{t} \quad \text { and } \quad \sigma_{R}^{(i) 2}=\sigma_{D}^{(i) 2}+\sigma_{F}^{2}+\sigma_{\tilde{P}}^{2}
$$

where $\left.\sigma_{D}^{(i)}\right)_{2}=\mathrm{E}_{\mathrm{t}}^{(i)}\left[G_{t}^{2}\right]$ and $\sigma_{\tilde{P}}^{2}=a^{2}\left(\sigma_{G}^{2}+b^{2} \sigma_{z}^{2}\right)$. Note that $\mathrm{E}_{\mathrm{t}}^{(1)}\left[G_{t}\right]=G_{t}, \mathrm{E}_{\mathrm{t}}^{(2)}\left[G_{t}\right]=$ $\gamma\left(G_{t}-b Z_{t}\right), \sigma_{D D}^{(1)}=0$, and $\sigma_{D}^{(2) 2}=\gamma \sigma_{G}^{2}$, where $\gamma=\left(\sigma_{G}^{2}+b^{2} \sigma_{z}^{2}\right)^{-1} \sigma_{G}^{2}$. Investors' stock demands are:

$$
X_{t}^{(1)}=\left(G_{t}-\tilde{P}_{t}-\sigma_{D N} Z_{t}\right) / \sigma_{R}^{(1) 2} \text { and } X_{t}^{(2)}=\left[\gamma\left(G_{t}-b Z_{t}\right)-\tilde{P}_{t}\right] / \sigma_{R}^{(2) 2} .
$$

Market clearing requires that $0=\omega X_{t}^{(1)}+(1-\omega) X_{t}^{(2)}$. Substituting in the investors' stock demands, we have

$$
\begin{aligned}
& 0=\omega(1-a) / \sigma_{R}^{(1) 2}+(1-\omega)(\gamma-a) / \sigma_{R}^{(2) 2} \\
& 0=\omega\left(\sigma_{D N}-a b\right) / \sigma_{R}^{(1) 2}+(1-\omega)(\gamma-a) b / \sigma_{R}^{(2) 2} .
\end{aligned}
$$

We can immediately solve for $b: b=\lambda \sigma_{D N}$. Note that $\gamma$ and $\sigma_{D D}^{(i)}(i=1,2)$ depend only on $b$, not on $a$. We have the following equation for $a$ :

$$
0=\omega \sigma_{R}^{(2) 2}(1-a)+(1-\omega) \sigma_{R}^{(1) 2}(\gamma-a) .
$$

Reorganizing terms, we have

$$
0=f(a) \equiv\left[a^{2}\left(\sigma_{G}^{2}+b^{2} \sigma_{Z}^{2}\right)+\left(\sigma_{F}^{2}+\omega \gamma \sigma_{G}^{2}\right)\right](a-\bar{a})-\omega \gamma \sigma_{G}^{2}(1-\bar{a})
$$

where $\bar{a}=1-(1-\omega)(1-\gamma) \geq 0$. First, note that for $a<\bar{a}, f(\bar{a})<0$. Thus, $f(a)=0$ has no real roots less than $\bar{a}$. Second, note that $f(\bar{a}) \leq 0$ and $f(a) \rightarrow \infty$ as $a \rightarrow \infty$. Thus, $f(a)=0$ has a real root no less than $\bar{a}$. Third, $f^{\prime}(a)>0$ for $a>\bar{a}$. We conclude that $f(a)=0$ has a unique root, which is non-negative.

Proof of Proposition 2. Suppose that $x, y, z$ are jointly normally distributed with zero means and a covariance matrix of $\Sigma$ where

$$
\Sigma=\left(\begin{array}{lll}
\sigma_{x x} & \sigma_{x y} & \sigma_{x z} \\
\sigma_{x y} & \sigma_{y y} & \sigma_{y z} \\
\sigma_{x z} & \sigma_{y z} & \sigma_{z z}
\end{array}\right) \equiv\left(\begin{array}{cc}
\Sigma_{11} & \Sigma_{12} \\
\Sigma_{12}^{\prime} & \Sigma_{22}
\end{array}\right)
$$


$\Sigma_{11}=\sigma_{x x}, \Sigma_{12}=\left(\sigma_{x y}, \sigma_{x z}\right)$, and $\Sigma_{22}=\left(\left(\sigma_{y y}, \sigma_{y z}\right) ;\left(\sigma_{y z}, \sigma_{z z}\right)\right)$. Then, we have $\mathrm{E}[x \mid y, z]=\beta_{x y} y+\beta_{x z} z$

where $\beta_{x y}=\left(\Sigma_{12} \Sigma_{22}^{-1}\right)_{1}$ and $\beta_{x z}=\left(\Sigma_{12} \Sigma_{22}^{-1}\right)_{2}$. Let $f_{+} \equiv \exp \left\{-\left(\Sigma_{22}^{-1}\right)_{y z} y|z|\right\}$ and $f_{-} \equiv \exp \left\{+\left(\Sigma_{22}^{-1}\right)_{y z} y|z|\right\}$. Then

$$
\begin{aligned}
\mathrm{E}[x|y,| z \mid] & =\beta_{x y} y+\frac{f_{+}-f_{-}}{f_{+}+f_{-}} \beta_{x z}|z|=\beta_{x y} y-\beta_{x z}|z| \tanh \left[\left(\Sigma_{22}^{-1}\right)_{y z}|z| y\right] \\
& \approx \beta_{x y} y-\beta_{x z}\left(\Sigma_{22}^{-1}\right)_{y z}|z|^{2} y .
\end{aligned}
$$

For our purpose, let $x=R_{t+1}, y=R_{t}$ and $z=\tilde{P}_{t}-\tilde{P}_{t-1}$. Then, $\sigma_{x x}=\sigma_{y y}=(1-a)^{2} \sigma_{G}^{2}+$ $a^{2} \sigma_{D N}^{2} \sigma_{Z}^{2}+\sigma_{R}^{2}, \sigma_{z z}=2 \sigma_{\tilde{P}}^{2}, \sigma_{x y}=a \sigma_{G}^{2}-\sigma_{\tilde{P}}^{2}, \sigma_{x z}=a \sigma_{G}^{2}-\sigma_{\tilde{P}}^{2}$, and $\sigma_{y z}=2 \sigma_{\tilde{P}}^{2}-a \sigma_{G}^{2}$, where $\sigma_{R}^{2}=\sigma_{F}^{2}+\sigma_{\tilde{P}}^{2}$. Then, we have

$$
\begin{aligned}
\mathrm{E}\left[R_{t+1}\left|R_{t},\right| \tilde{P}_{t}-\tilde{P}_{t-1} \mid\right] & =\beta_{1} R_{t}-\beta_{2}\left|\tilde{P}_{t}-\tilde{P}_{t-1}\right| \tanh \left(\eta\left|\tilde{P}_{t}-\tilde{P}_{t-1}\right| R_{t}\right) \\
& \approx\left(\theta_{1}-\theta_{2}^{\prime}\left|\tilde{P}_{t}-\tilde{P}_{t-1}\right|^{2}\right) R_{t}
\end{aligned}
$$

where

$$
\begin{aligned}
\beta_{1} & =\beta_{x y}=-\frac{a \sigma_{G}^{2}\left(\sigma_{\tilde{P}}^{2}-a \sigma_{G}^{2}\right)}{2 \sigma_{D}^{2} \sigma_{\tilde{P}}^{2}-a^{2} \sigma_{G}^{4}} \\
\beta_{2} & =\frac{\left(\sigma_{D}^{2}-a \sigma_{G}^{2}\right)\left(\sigma_{\tilde{P}}^{2}-a \sigma_{G}^{2}\right)}{2 \sigma_{D}^{2} \sigma_{\tilde{P}}^{2}-a^{2} \sigma_{G}^{4}} \\
\eta & =\frac{2 \sigma_{\tilde{P}}^{2}-a \sigma_{G}^{2}}{2 \sigma_{D}^{2} \sigma_{\tilde{P}}^{2}-a^{2} \sigma_{G}^{4}} \\
\theta_{1} & =\beta_{1} \\
\theta_{2}^{\prime} & =\beta_{2} \eta
\end{aligned}
$$

where $\sigma_{D}^{2}=\sigma_{F}^{2}+\sigma_{G}^{2}$. Since $\sigma_{\tilde{P}}^{2}=a\left(\sigma_{G}^{2}+b^{2} \sigma_{z}^{2}\right)(a-\gamma)$ and $a \geq \bar{a} \geq \gamma$, we have $\beta_{2} \geq 0$ and $\eta \geq 0$. Further note that $\left|\tilde{P}_{t}-\tilde{P}_{t-1}\right|=\sqrt{(2 / \pi)} \sigma_{\tilde{P}} \tilde{V}_{t}$. Letting $\theta_{2}=2 \sigma_{\tilde{P}}^{2} \theta_{2}^{\prime} / \pi$, we obtain (8).

Proof of Proposition 3. For $\sigma_{G}^{2}=0, a=\omega, \theta_{2}^{\prime}=1 /\left(2 \sigma_{D}^{2}\right)$, and

$$
\theta_{2}=\theta_{20}=\frac{\sigma_{\tilde{P}}^{2}}{\pi \sigma_{D}^{2}} \equiv \frac{\omega^{2}}{\pi} \frac{\sigma_{D N}^{2} \sigma_{Z}^{2}}{\sigma_{D}^{2}}
$$

For $\sigma_{G}^{2}$ small and holding $\sigma_{D}^{2}, \sigma_{\tilde{P}}^{2}$ constant, we have

$$
\theta_{2} \approx \frac{1}{\pi} \frac{\sigma_{D}^{2}}{\sigma_{\tilde{P}}^{2}}\left[1-a\left(\frac{\sigma_{G}^{2}}{\sigma_{D}^{2}}+\frac{3}{2} \frac{\sigma_{G}^{2}}{\sigma_{\tilde{P}}^{2}}\right)\right]+o\left(\sigma_{G}^{2}\right) .
$$

Realizing that $a=\omega+o\left(\sigma_{G}^{2}\right)$, we obtain Equation (9). 


\section{References}

Ajinkya B.B., and P.C. Jain, 1989, The Behavior of Daily Stock Market Trading Volume, Journal of Accounting and Economics 11, 331-359.

Antoniewicz, R.L., 1993, Relative Volume and Subsequent Stock Price Movements, working paper, Board of Governors of the Federal Reserve System.

Bhattacharya, U. and M. Spiegel, 1991, Insiders, Outsiders, and Market Breakdowns, Review of Financial Studies 4, 255-282.

Blume, L., D. Easley, and M. O'Hara, 1994, Market Statistics and Technical Analysis: The Role of Volume, Journal of Finance 49, 153-181.

Brennan, M. and A. Subrahmanyam, 1995, Investment Analysis and Price Formation in Securities Markets, Journal of Financial Economics 38, 361381.

Breusch, T.S., 1978, Testing for Autocorrelation in Dynamic Linear Models, Australian Economic Papers 17, 334-355.

Brown, D.P., and R.H. Jennings, 1989, On Technical Analysis, Review of Financial Studies 2, 527-551.

Campbell, J.Y., S.J. Grossman, and J. Wang, 1993, Trading Volume and Serial Correlation in Stock Returns, Quarterly Journal of Economics 108, 905-939.

Canina, L., R. Michaely, R. Thaler, and K. Womack, 1998, Caveat Compounder: A Warning about Using the Daily CRSP Equal-Weighted Index to Compute Long-Run Excess Returns, Journal of Finance 53, 403-416.

Conrad, J., A. Hameed, and Niden C.M., 1992, Volume and Autocovariances in Short-Horizon Individual Security Returns, Journal of Finance 49, 13051329 .

Conrad, J., G. Kaul, and M. Nimalendran, 1991, Components of Short-Horizon Individual Security Returns, Journal of Financial Economics 29, 365-384.

Cready, W.M., and Ramanan R., 1991, The Power of Tests Employing LogTransformed Volume in Detecting Abnormal Trading, Journal of Accounting and Economics 14, 203-214. 
Duffee, G., 1992, Trading volume and Return Reversals, Finance and Economics Discussion Paper Series No. 192, Board of Governors of the Federal Reserve System.

Easley, D., and M. O'Hara, 1987, Price, Trade Size, and Information In Securities Markets, Journal of Financial Economics 18, 69-90.

Easley, D., M. O'Hara, and J. Paperman, 1998, Are Financial Analysts Informed Traders? Journal of Financial Markets 1, 175-201.

French, K.R., and R. Roll, 1986, Stock Return Variances: The Arrival of Information and the Reaction of Traders, Journal of Financial Economics $17,5-26$.

Gallant, R., P. Rossi, and Tauchen, G., 1992, Stock Prices and Volume, Review of Financial Studies 5, 199-242.

Glosten, L. and P. Milgrom, 1985, Bid, Ask and Transaction Prices in a Market-Maker Market with Heterogeneously Informed Traders, Journal of Financial Economics 14, 71-100.

Godfrey, L.G., 1978a, Testing Against General Autoregressive and Moving Average Error Models when the Regressors Include Lagged Dependent Variables, Econometrica 46, 1293-1302.

Godfrey, L.G., 1978b, Testing Against Higher Order Serial Correlation in Regression Equations when the Regressors Include Lagged Dependent Variables, Econometrica 46, 1303-1310.

Grundy, B., and M. McNichols, 1989, Trade and Revelation of Information through Prices and Direct Disclosure, Review of Financial Studies 2, 495526 .

Hasbrouck, J., 1988, Trades, Quotes, Inventories, and Information, Journal of Financial Economics 22, 229-252.

Hasbrouck, J., 1991, Measuring the Information Content of Stock Trades, Journal of Finance 46, 179-207.

He, H., and J. Wang, 1995, Differential Information and Dynamic Behavior of Trading Volume, Review of Financial Studies 8, 919-972.

Jegadeesh, N., and S. Titman, 1995, Short-Horizon Return Reversals and the Bid-Ask Spread Journal of Financial Intermediation 4, 116-132. 
Johnston, J., 1985, Econometric Methods 3rd Edition, McGraw-Hill Book Company.

Jorion, P., 1990, The Exchange-Rate Exposure of U.S. Multinationals, Journal of Business 63, 331-345.

Kyle, Albert S.,1985, Continuous Auctions and Insider Trading, Econometrica $53,1315-1335$.

LeBaron, B., 1992, Persistence of the Dow Jones Index on Rising Volume, working paper, University of Wisconsin.

Lee, C.M.C., B. Mucklow, and M.J. Ready, 1993, Spreads, Depths, and the Impact of Earnings Information: an Intraday Analysis, Review of Financial Studies 6, 345-374.

Lee, C.M.C. and B. Swaminathan, 2000, Price Momentum and Trading Volume, forthcoming, Journal of Finance.

Lo, A.W., and A.C. MacKinlay, 1988, Stock Market Prices Do Not Follow Random Walks: Evidence from a Simple Specification Test, Review of Financial Studies 1, 41-66.

Lo, A.W., and A.C. MacKinlay, 1990, An Econometric Analysis of Nonsynchronous Trading, Journal of Econometrics 45, 181-211.

Lo, A. and J. Wang, 2000, Trading Volume: Definitions, Data Analysis, and Implications of Portfolio Theory, Review of Financial Studies 13, 257-300.

Madhavan, A., M. Richardson, and M. Roomans, 1997, Why Do Security Prices Change? A Transaction-Level Analysis of NYSE Stocks, Review of Financial Studies 10, 1035-1064.

Morse, D., 1980, Asymmetric Information in Securities Markets and Trading Volume, Journal of Financial and Quantitative Analysis 15, 1129-1148.

Richardson G., S.E. Sefcik, and R. Thompson, 1986, A Test of Dividend Irrelevance Using Volume Reactions to a Change in Dividend Policy, Journal of Financial Economics 17, 313-333.

Rousseeuw, P.J, and A.M. Leroy, 1987, Robust Regression and Outlier Detection, John Wiley \& Sons. 
Stickel, S.E. and R.E. Verrecchia, 1994, Evidence that Volume Sustains Price Changes, Financial Analyst Journal (November-December), 57-67.

Wang, J., 1994, A Model of Competitive Stock Trading Volume, Journal of Political Economy 102, 127-168. 


\section{Table 1 \\ Summary Statistics}

The sample contains 2,226 stocks. Included in the sample are all common stocks with information in the CRSP database that traded on the NYSE or AMEX between 1993 and 1998 and that had at least 1,000 days with trading. Panel A presents descriptive statistics by size groups. For each firm, AvgCap is the average daily market capitalization (number of shares outstanding multiplied by the daily closing price). AvgTrd is the average number of shares traded daily. AvgTurn $n_{\mathrm{i}}$ is the average daily turnover (number of shares traded divided by the number of shares outstanding), and $A v g \operatorname{Prc}_{i}$ is the average price of a firm's stock over the sample period. BAsprd ${ }_{i}$ is the average daily opening percentage spread (opening bid-ask spread divided by the opening midquote) over the sample period from the TAQ database. Panel B presents descriptive statistics for the volume series used in the time-series regressions. We define the daily volume series of a stock as the detrended, log-transformed daily turnover series. We report the averages for the entire sample and for three size groups of the mean, standard deviation, and selected autocorrelation coefficients of the volume series of the individual stocks.

Panel A: Characteristics of the Entire Sample and Three Size-Based Subsamples

\begin{tabular}{l|l|rrrrr}
\multicolumn{2}{l|}{} & $\begin{array}{r}\text { AvgCap } \\
\text { (in million \$) }\end{array}$ & $\begin{array}{c}\text { AvgTrd } \\
\text { (in 100s) }\end{array}$ & $\begin{array}{c}\text { AvgTurn } \\
\text { (in \%) }\end{array}$ & $\begin{array}{c}\text { AvgPrc } \\
\text { (in \$) }\end{array}$ & $\begin{array}{c}\text { BAsprd } \\
\text { (in \%) }\end{array}$ \\
\hline Entire Sample & Mean & $2,587.55$ & 1,997 & 0.321 & 25.59 & 2.19 \\
& Median & 473.52 & 672 & 0.258 & 22.40 & 1.38 \\
& Std. Dev & $8,019.00$ & 3,844 & 0.255 & 20.49 & 2.33 \\
& Minimum & 3.61 & 2 & 0.007 & 0.39 & 0.25 \\
& Maximum & $147,817.21$ & 52,735 & 2.837 & 330.45 & 19.51 \\
& Observations & 2226 & 2226 & 2226 & 2225 & 2225 \\
\hline Size Group: & Mean & 85.97 & 288 & 0.270 & 10.84 & 4.11 \\
Small & Median & 75.96 & 164 & 0.212 & 8.90 & 3.12 \\
& Std. Dev & 58.18 & 408 & 0.213 & 7.79 & 2.99 \\
& Observations & 742 & 742 & 742 & 742 & 741 \\
\hline Size Group: & Mean & 525.98 & 918 & 0.338 & 23.93 & 1.62 \\
Medium & Median & 473.52 & 601 & 0.273 & 22.91 & 1.35 \\
& Std. Dev & 237.23 & 994 & 0.263 & 11.24 & 1.13 \\
& Observations & 742 & 742 & 742 & 742 & 742 \\
\hline Size Group: & Mean & $7,150.69$ & 4,786 & 0.355 & 42.01 & 0.84 \\
Large & Median & $3,111.11$ & 2,863 & 0.291 & 37.20 & 0.75 \\
& Std. Dev & $12,714.35$ & 5,597 & 0.277 & 24.17 & 0.48 \\
& Observations & 742 & 742 & 742 & 741 & 742 \\
\hline
\end{tabular}

Panel B: Descriptive Statistics of the Volume (Detrended Log Turnover) Series

\begin{tabular}{l|cccccr} 
& Mean & $\begin{array}{c}\text { Standard } \\
\text { Deviation }\end{array}$ & $\begin{array}{c}\text { First } \\
\text { Autocorrelation AutocorrelationAutocorrelation }\end{array}$ & \# of stocks \\
\hline Entire Sample & 0.028 & 1.068 & 0.370 & 0.193 & 0.143 & 2226 \\
Size group: Low & 0.013 & 1.537 & 0.313 & 0.182 & 0.138 & 742 \\
Size group: Medium & 0.038 & 1.000 & 0.366 & 0.196 & 0.148 & 742 \\
Size group: High & 0.034 & 0.668 & 0.430 & 0.200 & 0.144 & 742
\end{tabular}




\section{Table 2 \\ Bid-Ask Spread and the Influence of Volume on the Autocorrelation of Returns}

This table shows the relation between information asymmetry and the influence of volume on the autocorrelation of stock returns. We use the average daily opening percentage spread of a stock over the sample period $\left(\right.$ BAsprd $\left._{\mathrm{i}}\right)$ as a proxy for information asymmetry. For each stock we measure the influence of volume on the autocorrelation of stock returns by the parameter $\mathrm{C} 2_{\mathrm{i}}$ from the following regression:

Return $_{i, t+1}=\mathrm{CO}_{\mathrm{i}}+\mathrm{Cl}_{\mathrm{i}} *$ Return $_{\mathrm{t}}+\mathrm{C}_{\mathrm{i}} *$ Volume $_{\mathrm{i}, \mathrm{t}} *$ Return $_{\mathrm{i}, \mathrm{t}}+$ error $_{\mathrm{i}, \mathrm{t}+1}$

where Volume $_{i, t}$ is the daily detrended log turnover of an individual stock and Return $n_{i, t}$ is the daily return of an individual stock in the CRSP database. In Panel A, we report the mean value of each parameter for three groups (Low, Medium, and High) of the information asymmetry proxy (BAsprd). We also note the number of negative parameters and the number of statistically significant (at the $10 \%$ level) parameters.

In Panel B, we provide an analogous analysis using the following cross-sectional regression:

$$
\mathrm{C}_{\mathrm{i}}=\mathrm{a}+\mathrm{b}^{*} \mathrm{ORDBA}_{\mathrm{i}}+\mathrm{ERROR}_{\mathrm{i}}
$$

where ORDBA is a variable representing the ordinal scale of BAsprd. T-statistics appear in parentheses.

Panel A: Categorical Analysis

\begin{tabular}{|c|c|c|c|c|c|c|c|c|}
\hline & $\begin{array}{c}\mathrm{C} 0 \\
\#<0\end{array}$ & $\begin{array}{c}\mathrm{C} 1 \\
\#<0\end{array}$ & $\begin{array}{c}\mathrm{C} 2 \\
\#<0\end{array}$ & $\begin{array}{c}\mathrm{t}_{\mathrm{C} 0} \\
|\#|>1.64\end{array}$ & $\begin{array}{c}\mathrm{t}_{\mathrm{C} 1} \\
|\#|>1.64\end{array}$ & $\begin{array}{c}\mathrm{t}_{\mathrm{C} 2} \\
|\#|>1.64\end{array}$ & $\mathrm{R}^{2}(\%)$ & $\begin{array}{c}\text { BAsprd } \\
\text { (in } \% \text { ) }\end{array}$ \\
\hline Low & 0.000770 & 0.013323 & -0.002814 & 1.698 & 0.336 & -0.096 & 0.564 & 0.719 \\
\hline$n=742$ & 30 & 328 & 378 & 409 & 337 & 240 & & \\
\hline Medium & 0.000722 & 0.010931 & 0.003168 & 1.239 & 0.188 & 0.232 & 0.921 & 1.417 \\
\hline $\mathrm{n}=742$ & 74 & 326 & 357 & 250 & 431 & 257 & & \\
\hline High & 0.000740 & -0.120346 & 0.035495 & 0.709 & -4.003 & 2.066 & 2.754 & 4.428 \\
\hline$n=742$ & 167 & 609 & 141 & 135 & 548 & 438 & & \\
\hline
\end{tabular}

Panel B: Regression Analysis

\begin{tabular}{lrrrr}
\hline Dependent variable & $\mathrm{a}$ & $\mathrm{b}$ & $\mathrm{R}^{2}(\%)$ & Observations \\
\hline $\mathrm{C} 2$ & -0.015921 & 0.055716 & 10.197 & 2,226 \\
& $(-7.863)$ & $(15.891)$ & & \\
\hline
\end{tabular}




\section{Table 3 \\ Market Capitalization and the Influence of Volume on the Autocorrelation of Returns}

This table shows the relation between information asymmetry and the influence of volume on the autocorrelation of stock returns. We use the average market capitalization of a firm over the sample period (AvgCap $\sin _{\mathrm{i}}$ a proxy for information asymmetry. For each stock we measure the influence of volume on the autocorrelation of stock returns by the parameter $\mathrm{C} 2{ }_{\mathrm{i}}$ from the following regression:

$$
\text { Return }_{i, t+1}=\mathrm{CO}_{\mathrm{i}}+\mathrm{Cl}_{\mathrm{i}} * \text { Return }_{\mathrm{t}}+\mathrm{C}_{\mathrm{i}} * \text { Volume }_{\mathrm{i}, \mathrm{t}} * \text { Return }_{\mathrm{i}, \mathrm{t}}+\text { error }_{\mathrm{i}, \mathrm{t}+1}
$$

where Volume $_{i, t}$ is the daily detrended log turnover of an individual stock and Return $n_{i, t}$ is the daily return of an individual stock in the CRSP database. In Panel A, we report the mean value of each parameter for three groups (Low, Medium, and High) of the information asymmetry proxy (AvgCap). We also note the number of negative parameters and the number of statistically significant (at the $10 \%$ level) parameters. In Panel B, we provide an analogous analysis using the following cross-sectional regression:

$$
\mathrm{C} 2_{\mathrm{i}}=\mathrm{a}+\mathrm{b}^{*} \mathrm{ORDCAP}_{\mathrm{i}}+\mathrm{ERROR}_{\mathrm{i}}
$$

where ORDCAP is a variable representing the ordinal scale of AvgCap. T-statistics appear in parentheses.

\begin{tabular}{|c|c|c|c|c|c|c|c|c|}
\hline & $\begin{array}{c}\mathrm{C} 0 \\
\#<0\end{array}$ & $\begin{array}{c}\mathrm{C} 1 \\
\#<0\end{array}$ & $\begin{array}{c}\mathrm{C} 2 \\
\#<0\end{array}$ & $\begin{array}{c}\mathrm{t}_{\mathrm{C} 0} \\
|\#|>1.64\end{array}$ & $\begin{array}{c}t_{\mathrm{C} 1} \\
|\#|>1.64\end{array}$ & $\begin{array}{c}\mathrm{t}_{\mathrm{C} 2} \\
|\#|>1.64\end{array}$ & $\mathrm{R}^{2}(\%)$ & $\begin{array}{c}\text { AvgCap } \\
\text { (in million } \$ \text { ) }\end{array}$ \\
\hline Low & 0.000784 & -0.104234 & 0.030277 & 0.843 & -3.538 & 1.848 & 2.729 & 85.97 \\
\hline$n=742$ & 139 & 557 & 167 & 157 & 550 & 429 & & \\
\hline Medium & 0.000672 & 0.005492 & 0.004852 & 1.178 & 0.028 & 0.289 & 0.996 & 525.98 \\
\hline $\mathrm{n}=742$ & 95 & 348 & 355 & 258 & 433 & 284 & & \\
\hline High & 0.000776 & 0.002650 & 0.000719 & 1.625 & 0.032 & 0.065 & 0.514 & 7150.69 \\
\hline$n=742$ & 37 & 358 & 354 & 379 & 333 & 222 & & \\
\hline
\end{tabular}

Panel A: Categorical Analysis

Panel B: Regression Analysis

\begin{tabular}{lccrr}
\hline Dependent variable & $\mathrm{a}$ & $\mathrm{b}$ & $\mathrm{R}^{2}(\%)$ & Observations \\
\hline $\mathrm{C} 2$ & 0.034081 & -0.044242 & 6.430 & 2,226 \\
& $(16.488)$ & $(-12.362)$ & & \\
\hline
\end{tabular}




\section{Table 4}

\section{Alternative Econometric Specifications: Autoregressive Structure}

For each stock, we measure the influence of volume on the autocorrelation of stock returns by the parameter $\mathrm{C}_{2} \mathrm{i}$ from the following regression:

$$
\text { Return }_{\mathrm{i}, \mathrm{t}+1}=\mathrm{C}_{\mathrm{i}}+\mathrm{Cl}_{\mathrm{i}} * \text { Return }_{\mathrm{t}}+\mathrm{C}_{\mathrm{i}} * \text { Volume }_{\mathrm{i}, \mathrm{t}} * \text { Return }_{\mathrm{i}, \mathrm{t}}+\text { error }_{\mathrm{i}, \mathrm{t}+1}
$$

where Volume $e_{i, t}$ is the daily detrended log turnover of an individual stock and Return ${ }_{i, t}$ is the daily return of an individual stock in the CRSP database. For each stock, we test for white noise against the alternative of an autoregressive error structure of orders one through five using the Breusch-Godfrey test. We use a 5\% significance level to reject the white noise hypothesis. In Panel A, we identify the most appropriate autoregressive structure using Algorithm A as follows: If the test is significant for any order $p<5$ but not for higher orders, we test again with the null of $\mathrm{AR}(\mathrm{p})$ against an autoregressive structure of orders higher than $\mathrm{p}$, but only up to order five. After identifying the appropriate order, we estimate the above relation using maximum likelihood with the suitable autoregressive structure. In Panel B, we identify the autoregressive structure using Algorithm B, which uses only the white noise test against an AR structure for each individual stock. Both panels report the results of the cross-sectional regressions:

$$
\begin{array}{r}
\mathrm{C}_{\mathrm{i}}=\mathrm{a}+\mathrm{b}^{*} \text { ORDBA }_{\mathrm{i}}+\mathrm{ERROR}_{\mathrm{i}} \\
\mathrm{C}_{\mathrm{i}}=\mathrm{a}+\mathrm{b}^{*} \text { ORDCAP }_{\mathrm{i}}+\text { ERROR }_{\mathrm{i}}
\end{array}
$$

where ORDBA and ORDCAP are variables representing the ordinal scales of BAsprd and AvgCap, respectively. Tstatistics appear in parentheses.

Panel A: Autoregressive Structure Identified Using Algorithm A $\mathrm{C} 2_{\mathrm{i}}=\mathrm{a}+\mathrm{b} * \mathrm{ORDBA}_{\mathrm{i}}+\mathrm{ERROR}_{\mathrm{i}}$

\begin{tabular}{rrrr}
\hline $\mathrm{a}$ & $\mathrm{b}$ & $\mathrm{R}^{2}(\%)$ & Observations \\
\hline-0.015298 & 0.060185 & 10.339 & 2,226 \\
$(-7.048)$ & $(16.014)$ & & \\
\hline
\end{tabular}

\begin{tabular}{ccrr}
$\mathrm{C}_{2}=\mathrm{a}+\mathrm{b}^{*}$ ORDCAP $_{\mathrm{i}}+$ ERROR $_{\mathrm{i}}$ & & \\
\hline $\mathrm{a}$ & $\mathrm{b}$ & $\mathrm{R}^{2}(\%)$ & Observations \\
\hline 0.038386 & -0.047135 & 6.341 & 2,226 \\
$(17.303)$ & $(-12.271)$ & & \\
& & & \\
\end{tabular}

Panel B: Autoregre ssive Structure Identified Using Algorithm B $\mathrm{C} 2_{\mathrm{i}}=\mathrm{a}+\mathrm{b}^{*} \mathrm{ORDBA}_{\mathrm{i}}+\mathrm{ERROR}_{\mathrm{i}}$

\begin{tabular}{rrrr}
\hline $\mathrm{a}$ & $\mathrm{B}$ & $\mathrm{R}^{2}(\%)$ & Observations \\
\hline-0.015485 & 0.060403 & 10.459 & 2,226 \\
$(-7.154)$ & $(16.118)$ & & \\
\hline
\end{tabular}

\begin{tabular}{rrrr}
$\mathrm{C}_{\mathrm{i}}=\mathrm{a}+\mathrm{b}^{*}$ ORDCAP $_{\mathrm{i}}+$ ERROR $_{\mathrm{i}}$ & & \\
\hline $\mathrm{a}$ & $\mathrm{b}$ & $\mathrm{R}^{2}(\%)$ & Observations \\
\hline 0.038443 & -0.047403 & 6.442 & 2,226 \\
$(17.376)$ & $(-12.374)$ & & \\
\hline
\end{tabular}




\section{Table 5}

\section{Alternative Econometric Specifications: Sensitivity to Common Factor}

For each stock, we measure the influence of volume on the autocorrelation of stock returns by the parameter D2 ${ }_{\mathrm{i}}$ from the following time-series regression:

$$
\operatorname{Return}_{\mathrm{i}, \mathrm{t}+1}=\mathrm{D} 0_{\mathrm{i}}+\mathrm{D} 1_{\mathrm{i}} * \operatorname{Return}_{\mathrm{t}}+\mathrm{D} 2_{\mathrm{i}} * \text { Volume }_{\mathrm{i}, \mathrm{t}} * \operatorname{Return}_{\mathrm{i}, \mathrm{t}}+\mathrm{D} 3 * \mathrm{MktRet}_{\mathrm{t}+1}+\text { error }_{\mathrm{i}, \mathrm{t}+1}
$$

MktRet $_{t+1}$ is the return on a value-weighted portfolio comprised of all common stocks that traded on the NYSE or AMEX that have valid return and volume information in the CRSP database for that day. The incorporation of MktRet $_{t+1}$ in the time-series regressions is intended to reduce potential correlation of the errors across stocks that arises from sensitivity to common factors. We report the results of the cross-sectional regressions:

$$
\begin{aligned}
& \mathrm{D} 2_{\mathrm{i}}=\mathrm{a}+\mathrm{b}^{*} \mathrm{ORDBA}_{\mathrm{i}}+\mathrm{ERROR}_{\mathrm{i}} \\
& \mathrm{D} 2_{\mathrm{i}}=\mathrm{a}+\mathrm{b}^{*} \mathrm{ORDCAP}_{\mathrm{i}}+\mathrm{ERROR}_{\mathrm{i}}
\end{aligned}
$$

where ORDBA and ORDCAP are variables representing the ordinal scales of BAsprd and AvgCap, respectively. Tstatistics appear in parentheses.

$\mathrm{D} 2_{\mathrm{i}}=\mathrm{a}+\mathrm{b}^{*} \mathrm{ORDBA}_{\mathrm{i}}+\mathrm{ERROR}_{\mathrm{i}}$

\begin{tabular}{rrrr}
\hline $\mathrm{a}$ & $\mathrm{b}$ & $\mathrm{R}^{2}(\%)$ & Observations \\
\hline-0.005528 & 0.043256 & 6.693 & 2,226 \\
$(-2.795)$ & $(12.631)$ & & \\
\hline
\end{tabular}

\begin{tabular}{crrr}
$\mathrm{D} 2$ & $\mathrm{i}=\mathrm{a}+\mathrm{b} *$ ORDCAP $_{\mathrm{i}}+$ ERROR $_{\mathrm{i}}$ & & \\
\hline $\mathrm{a}$ & \multicolumn{1}{c}{$\mathrm{b}$} & $\mathrm{R}^{2}(\%)$ & Observations \\
\hline 0.031677 & -0.031121 & 3.465 & 2,226 \\
$(15.745)$ & $(-8.934)$ & &
\end{tabular}




\section{Table 6}

\section{Alternative Econometric Specifications: Least Trimmed Squares}

For each stock, we measure the influence of volume on the autocorrelation of stock returns by the parameter $\mathrm{C} 2_{\mathrm{i}}$ from the following regression:

$$
\text { Return }_{i, t+1}=\mathrm{CO}_{\mathrm{i}}+\mathrm{Cl}_{\mathrm{i}} * \text { Return }_{\mathrm{t}}+\mathrm{C}_{\mathrm{i}} * \text { Volume }_{\mathrm{i}, \mathrm{t}} * \text { Return }_{\mathrm{i}, \mathrm{t}}+\text { error }_{\mathrm{i}, \mathrm{t}+1}
$$

${\text { where } \text { Volume }_{i, t} \text { is the daily detrended log turnover of an individual stock and Return }}_{i, t}$ is the daily return of an individual stock in the CRSP database. We estimate this relation in two stages. In the first stage, we use a least trimmed squares (LTS) estimator to identify outliers (by minimizing the sum of the h smallest squared residuals, where $h=2 / 3$ of the observations for each stock). In the second stage, we use a weighted least squares (WLS) estimation in which we make the weights on the outliers identified in the first stage equal to zero. The $\mathrm{C} 2_{\mathrm{i}}$ parameters for the cross-sectional estimation are taken from the WLS estimation. In Panel A, we report the results of the OLS cross-sectional regressions:

$$
\begin{gathered}
\mathrm{C}_{2}=\mathrm{a}+\mathrm{b}^{*} \mathrm{ORDBA}_{\mathrm{i}}+\mathrm{ERROR}_{\mathrm{i}} \\
\mathrm{C} 2_{\mathrm{i}}=\mathrm{a}+\mathrm{b}^{*} \text { ORDCAP }_{\mathrm{i}}+\text { ERROR }_{\mathrm{i}}
\end{gathered}
$$

where ORDBA and ORDCAP are variables representing the ordinal scales of BAsprd and AvgCap, respectively. In Panel B, we apply the two-stage LTS estimation to the cross-sectional, rather than the time-series, regressions. We take the $\mathrm{C} 2_{\mathrm{i}}$ coefficients from the OLS time series regressions that were presented in Tables 2 and 3, but we use LTS to estimate the relations with the information asymmetry proxies. We present the results of the second-stage WLS estimation. T-statistics appear in parentheses.

\begin{tabular}{|c|c|c|c|}
\hline $\mathrm{a}$ & $\mathrm{b}$ & $\mathrm{R}^{2}(\%)$ & Observations \\
\hline $\begin{array}{r}-0.011487 \\
(-5.483)\end{array}$ & $\begin{array}{r}0.049199 \\
(13.562)\end{array}$ & 7.638 & 2,226 \\
\hline \multicolumn{4}{|c|}{$\mathrm{C}_{2}=\mathrm{a}+\mathrm{b}^{*} \mathrm{ORDCAP}_{\mathrm{i}}+\mathrm{ERROR}_{\mathrm{i}}$} \\
\hline $\mathrm{a}$ & $\mathrm{b}$ & $\mathrm{R}^{2}(\%)$ & Observations \\
\hline $\begin{array}{r}0.032918 \\
(15.487)\end{array}$ & $\begin{array}{r}-0.039572 \\
(-10.752)\end{array}$ & 4.942 & 2,226 \\
\hline
\end{tabular}

Panel A: Two-Stage Least Trimmed Squares Applied to Time-Series Regressions $\mathrm{C}_{\mathrm{i}}=\mathrm{a}+\mathrm{b}^{*} \mathrm{ORDBA}_{\mathrm{i}}+\mathrm{ERROR}_{\mathrm{i}}$

Panel B: Two-Stage Least Trimmed Squares Applied to Cross-Sectional Regressions $\mathrm{C} 2_{\mathrm{i}}=\mathrm{a}+\mathrm{b}^{*} \mathrm{ORDBA}_{\mathrm{i}}+\mathrm{ERROR}_{\mathrm{i}}$

\begin{tabular}{rrrr}
\hline $\mathrm{a}$ & $\mathrm{B}$ & $\mathrm{R}^{2}(\%)$ & Observations \\
\hline-0.019349 & 0.060732 & 14.328 & 2,226 \\
$(-10.40)$ & $(19.00)$ & & \\
\hline
\end{tabular}

\begin{tabular}{rrrr}
$\mathrm{C}_{2} \mathrm{i}=\mathrm{a}+\mathrm{b}^{*}$ ORDCAP $_{\mathrm{i}}+$ ERROR $_{\mathrm{i}}$ & & \\
\hline $\mathrm{a}$ & $\mathrm{b}$ & $\mathrm{R}^{2}(\%)$ & Observations \\
\hline 0.034651 & -0.046424 & 8.586 & 2,226 \\
$(18.57)$ & $(-14.25)$ & & \\
& & & \\
\hline
\end{tabular}




\section{Table 7 \\ Alternative Lengths of Time Intervals}

Panel A replaces the daily interval for measuring return and volume with an alternative interval that allows for roughly the same level of noise trading across stocks. We use the median daily turnover (MedTurn) of a stock as a measure of the normal level of trading. We group all stocks into three categories (High, Medium and Low) according to their MedTurn. We use a daily interval for stocks in the High group, a two-day interval for stocks in the Medium group, and a five-day interval for stocks in the Low group (so that the average turnover across groups is equal). We calculate the return and turnover series for a stock in the medium and low groups by compounding daily returns and linearly adding turnover for the days in each interval. For each stock, we measure the influence of volume on the autocorrelation of stock returns by the parameter $\mathrm{C} 2_{\mathrm{i}}$ from the following regression:

$$
\text { Return }_{i, t+1}=\mathrm{CO}_{\mathrm{i}}+\mathrm{Cl}_{\mathrm{i}} * \text { Return }_{\mathrm{t}}+\mathrm{C}_{\mathrm{i}} * \text { Volume }_{\mathrm{i}, \mathrm{t}} * \text { Return }_{\mathrm{i}, \mathrm{t}}+\text { error }_{\mathrm{i}, \mathrm{t}+1}
$$

where Volume $_{i, t}$ is the detrended log turnover of an individual stock and Return ${ }_{i, t}$ is the return of an individual stock. We report the results of the OLS cross-sectional regressions:

$$
\begin{gathered}
\mathrm{C}_{\mathrm{i}}=\mathrm{a}+\mathrm{b}^{*} \mathrm{ORDBA}_{\mathrm{i}}+\mathrm{ERROR}_{\mathrm{i}} \\
\mathrm{C} 2_{\mathrm{i}}=\mathrm{a}+\mathrm{b}^{*} \mathrm{ORDCAP}_{\mathrm{i}}+\mathrm{ERROR}_{\mathrm{i}}
\end{gathered}
$$

where ORDBA and ORDCAP are variables representing the ordinal scales of BAsprd and AvgCap, respectively. In Panel B, we test the robustness of our results to a longer estimation period. We estimate the time series regressions for the stocks in our sample using 10 years of data (1989-1998). We present the results of the cross-sectional regressions using the same information asymmetry proxies as in Panel A. T-statistics appear in parentheses.

Panel A: Using an Alternative to the Daily Interval for Measuring Return and Volume $\mathrm{C} 2_{\mathrm{i}}=\mathrm{a}+\mathrm{b}^{*} \mathrm{ORDBA}_{\mathrm{i}}+\mathrm{ERROR}_{\mathrm{i}}$

\begin{tabular}{crrr}
\hline $\mathrm{a}$ & $\mathrm{B}$ & $\mathrm{R}^{2}(\%)$ & Observations \\
\hline-0.015534 & 0.038606 & 3.531 & 2,226 \\
$(-6.286)$ & $(9.023)$ & & \\
\hline \multicolumn{5}{c}{$2_{\mathrm{i}}=\mathrm{a}+\mathrm{b}^{*}$ ORDCAP $_{\mathrm{i}}+$ ERROR $_{\mathrm{i}}$} \\
$\mathrm{a}$ & $\mathrm{b}$ & $\mathrm{R}^{2}(\%)$ & Observations \\
\hline 0.016285 & -0.025003 & 1.481 & 2,226 \\
$(6.521)$ & $(-5.782)$ & & \\
\hline
\end{tabular}

Panel B: Using Ten Years of Data (1989-1998)

\begin{tabular}{rrrr}
$\mathrm{C}_{2} \mathrm{i}=\mathrm{a}+\mathrm{b}^{*}$ ORDBA $_{\mathrm{i}}+$ ERROR $_{\mathrm{i}}$ & & \\
\hline $\mathrm{a}$ & $\mathrm{B}$ & $\mathrm{R}^{2}(\%)$ & Observations \\
-0.014592 & 0.057887 & 13.461 & 2,226 \\
$(-8.118)$ & $(18.599)$ & & \\
\hline
\end{tabular}

\begin{tabular}{ccrr}
$\mathrm{C}_{2} \mathrm{i}=\mathrm{a}+\mathrm{b}^{*}$ ORDCAP $_{\mathrm{i}}+$ ERROR $_{\mathrm{i}}$ & & \\
\hline $\mathrm{a}$ & $\mathrm{b}$ & $\mathrm{R}^{2}(\%)$ & Observations \\
\hline 0.037995 & -0.047240 & 8.965 & 2,226 \\
$(20.609)$ & $(-14.799)$ & & \\
\hline
\end{tabular}




\section{Table 8 \\ Eliminating the Bid-Ask Bounce Effect}

The influence of volume on the autocorrelation of stock returns is measured by the parameter $\mathrm{C} 22_{\mathrm{i}}$ from the following time-series regression:

Return $_{\mathrm{i}, \mathrm{t}+1}=\mathrm{CO}_{\mathrm{i}}+\mathrm{Cl}_{\mathrm{i}} *$ Return $_{\mathrm{i}, \mathrm{t}}+\mathrm{C}_{\mathrm{i}} *$ Volume $_{\mathrm{i}, \mathrm{t}} *$ Return $_{\mathrm{i}, \mathrm{t}}+$ error $_{\mathrm{i}, \mathrm{t}+1}$

To eliminate the effects of bid-ask bounce, we calculate Return ${ }_{\mathrm{i}}$, from end-of-day mid-quotes. We report the results of the cross-sectional regressions:

$$
\begin{array}{r}
\mathrm{C}_{\mathrm{i}}=\mathrm{a}+\mathrm{b}^{*} \mathrm{ORDBA}_{\mathrm{i}}+\mathrm{ERROR}_{\mathrm{i}} \\
\mathrm{C}_{\mathrm{i}}=\mathrm{a}+\mathrm{b}^{*} \mathrm{ORDCAP}_{\mathrm{i}}+\mathrm{ERROR}_{\mathrm{i}}
\end{array}
$$

where ORDBA and ORDCAP are variables representing the ordinal scales of BAsprd and AvgCap, respectively. To eliminate the effects of outliers, the cross-sectional regressions are estimated using the two-stage least trimmed squares procedure described in Section 4.1 .

\begin{tabular}{rrrr}
$\mathrm{C}_{2} \mathrm{i}=\mathrm{a}+\mathrm{b}^{*}$ ORDBA $_{\mathrm{i}}+$ ERROR $_{\mathrm{i}}$ & & \\
\hline $\mathrm{a}$ & $\mathrm{b}$ & $\mathrm{R}^{2}(\%)$ & Observations \\
\hline-0.020033 & 0.028729 & 2.674 & 2,225 \\
$(-8.87)$ & $(7.45)$ & & \\
\hline
\end{tabular}

\begin{tabular}{rrrr}
$\mathrm{C}_{2}=\mathrm{a}+\mathrm{b}^{*}$ ORDCAP $_{\mathrm{i}}+$ ERROR $_{\mathrm{i}}$ \\
$\mathrm{a}$ & $\mathrm{b}$ & $\mathrm{R}^{2}(\%)$ & Observations \\
\hline 0.006676 & -0.025449 & 2.050 & 2,225 \\
$(3.01)$ & $(-6.52)$ & & \\
\hline
\end{tabular}




\section{Table 9}

\section{Alternative Definitions of Volume}

In Panel A, the influence of volume on the autocorrelation of stock returns is measured by the parameter $\mathrm{C} 2{ }_{\mathrm{i}}$ from the following time-series regression:

$$
\text { Return }_{i, t+1}=\mathrm{CO}_{\mathrm{i}}+\mathrm{Cl}_{\mathrm{i}} * \text { Return }_{\mathrm{t}}+\mathrm{C}_{\mathrm{i}} * \text { Volume }_{\mathrm{i}, \mathrm{t}} * \text { Return }_{\mathrm{i}, \mathrm{t}}+\text { error }_{\mathrm{i}, \mathrm{t}+1}
$$

where turnover (the daily number of shares traded divided by the number of shares outstanding) serves as the alternative definition of volume.

In Panel B, the influence of volume on the autocorrelation of stock returns is measured by the parameter $\mathrm{C} 2 \mathrm{i}_{\mathrm{i}}$ from the following time-series regression:

$$
\text { Return }_{\mathrm{i}, \mathrm{t}+1}=\mathrm{CO}_{\mathrm{i}}+\mathrm{Cl}_{\mathrm{i}} * \text { Return }_{\mathrm{t}}+\mathrm{C} 2_{\mathrm{i}} *\left(\text { Volume }_{\mathrm{i}, \mathrm{t}}\right)^{2} * \operatorname{Return}_{\mathrm{i}, \mathrm{t}}+\text { error }_{\mathrm{i}, \mathrm{t}+1}
$$

where $\log (1+$ daily number of shares traded $)$ serves as the alternative definition of volume.

Using the results of the time-series regressions, both panels report the results of the cross-sectional regressions:

$$
\begin{gathered}
C 2_{\mathrm{i}}=\mathrm{a}+\mathrm{b}^{*} \text { ORDBA }_{\mathrm{i}}+\mathrm{ERROR}_{\mathrm{i}} \\
\mathrm{C} 2_{\mathrm{i}}=\mathrm{a}+\mathrm{b}^{*} \text { ORDCAP }_{\mathrm{i}}+\mathrm{ERROR}_{\mathrm{i}}
\end{gathered}
$$

where ORDBA and ORDCAP are variables representing the ordinal scales of BAsprd and AvgCap, respectively. Tstatistics appear in parentheses.

Panel A: Volume Is Measured as Turnover

$\mathrm{C}_{\mathrm{i}}=\mathrm{a}+\mathrm{b}^{*} \mathrm{ORDBA}_{\mathrm{i}}+\mathrm{ERROR}_{\mathrm{i}}$

\begin{tabular}{rrrr}
\hline $\mathrm{a}$ & $\mathrm{b}$ & $\mathrm{R}^{2}(\%)$ & Observations \\
\hline-3.322436 & 10.685070 & 2.269 & 2,226 \\
$(-3.869)$ & $(7.186)$ & & \\
& & & \\
\hline
\end{tabular}

\begin{tabular}{rrrr}
$\mathrm{C}_{2} \mathrm{i}=\mathrm{a}+\mathrm{b}^{*}$ ORDCAP $_{\mathrm{i}}+$ ERROR $_{\mathrm{i}}$ & & \\
\hline $\mathrm{a}$ & $\mathrm{b}$ & $\mathrm{R}^{2}(\%)$ & Observations \\
\hline 6.408573 & -8.768206 & 1.528 & 2,226 \\
$(7.434)$ & $(-5.875)$ & & \\
\hline
\end{tabular}

Panel B: Volume Is Measured as $\log (1+$ Shares Traded $)$

$\mathrm{C}_{\mathrm{i}}=\mathrm{a}+\mathrm{b}^{*} \mathrm{ORDBA}_{\mathrm{i}}+\mathrm{ERROR}_{\mathrm{i}}$

\begin{tabular}{rrrr}
\hline $\mathrm{a}$ & $\mathrm{B}$ & $\mathrm{R}^{2}(\%)$ & Observations \\
\hline-0.001029 & 0.003526 & 19.999 & 2,226 \\
$(-11.917)$ & $(23.579)$ & & \\
\hline
\end{tabular}

\begin{tabular}{ccrr}
$\mathrm{C} 2_{\mathrm{i}}=\mathrm{a}+\mathrm{b}^{*}$ ORDCAP $_{\mathrm{i}}+$ ERROR $_{\mathrm{i}}$ & & \\
\hline $\mathrm{a}$ & $\mathrm{b}$ & $\mathrm{R}^{2}(\%)$ & Observations \\
\hline 0.002335 & -0.003199 & 16.461 & 2,226 \\
$(26.454)$ & $(-20.934)$ & &
\end{tabular}




\section{Table 10 \\ Analyst Following as an Information Asymmetry Proxy}

The influence of volume on the autocorrelation of stock returns is measured by the parameter $\mathrm{C} 2_{\mathrm{i}}$ from the following time-series regression:

$$
\text { Return }_{i, t+1}=\mathrm{CO}_{\mathrm{i}}+\mathrm{Cl}_{\mathrm{i}} * \text { Return }_{\mathrm{t}}+\mathrm{C} 2_{\mathrm{i}} * \text { Volume }_{\mathrm{i}, \mathrm{t}} * \text { Return }_{\mathrm{i}, \mathrm{t}}+\text { error }_{\mathrm{i}, \mathrm{t}+1}
$$

To construct the proxy for analyst following, we look for the monthly number of analysts who provide I/B/E/S with an end-of-fis cal-year earnings forecast for the current year. NUMEST $\mathrm{N}_{\mathrm{i}}$ is the average monthly number of analysts over the sample period (six years). We use this variable as a proxy for information asymmetry (the more analysts, the less information asymmetry). We use a reduced sample of 2035 firms for which we found information concerning analyst following in the $\mathrm{I} / \mathrm{B} / \mathrm{E} / \mathrm{S}$ database. We report the results of the cross-sectional regressions:

$$
\begin{aligned}
& \mathrm{C} 2_{\mathrm{i}}=\mathrm{a}+\mathrm{b}^{*} \mathrm{NUMEST}_{\mathrm{i}}+\mathrm{ERROR}_{\mathrm{i}} \\
& \mathrm{C} 2_{\mathrm{i}}=\mathrm{a}+\mathrm{b}^{*} \text { ORDEST }_{\mathrm{i}}+\text { ERROR }_{\mathrm{i}}
\end{aligned}
$$

ORDEST $_{i}$ is a variable representing the ordinal scale of NUMEST ${ }_{i}$, where two firms that have the same number of analysts receive the same rank. T-statistics appear in parentheses.

\begin{tabular}{rrrr}
$\mathrm{C}_{2} \mathrm{i}=\mathrm{a}+\mathrm{b}^{*}$ NUMEST $_{\mathrm{i}}+$ ERROR $_{\mathrm{i}}$ & & \\
\hline $\mathrm{a}$ & \multicolumn{1}{c}{$\mathrm{b}$} & $\mathrm{R}^{2}(\%)$ & Observations \\
\hline 0.016435 & -0.000760 & 1.395 & 2,035 \\
$(9.789)$ & $(-5.362)$ & &
\end{tabular}

\begin{tabular}{crrr}
$\mathrm{C}_{2} \mathrm{i}=\mathrm{a}+\mathrm{b}^{*}$ ORDEST $_{\mathrm{i}}+$ ERROR $_{\mathrm{i}}$ & & \\
\hline $\mathrm{a}$ & \multicolumn{1}{c}{$\mathrm{b}$} & $\mathrm{R}^{2}(\%)$ & Observations \\
\hline 0.022239 & -0.026790 & 2.611 & 2,035 \\
$(10.970)$ & $(-7.383)$ & &
\end{tabular}




\section{Table 11 \\ Firm-Specific Information Asymmetry and return-volume relation}

This table contains an empirical test of the hypothesis that firm-specific information asymmetry is a driving force behind the dynamic relation between returns and volume. For each stock, we measure the influence of volume on the autocorrelation of stock returns by the parameter $\mathrm{C} 2_{\mathrm{i}}$ from the following regression:

ResReturn $_{\mathrm{i}, \mathrm{t}+1}=\mathrm{CO}_{\mathrm{i}}+\mathrm{C1}_{\mathrm{i}} * \operatorname{ResReturn}_{\mathrm{i}, \mathrm{t}}+\mathrm{C}_{\mathrm{i}} * \operatorname{Res}_{\text {Volume }}{ }_{\mathrm{i}, \mathrm{t}} * \operatorname{ResReturn}_{\mathrm{i}, \mathrm{t}}+$ error $_{\mathrm{i}, \mathrm{t}+1}$

ResReturn $_{i, t}$ is the daily abnormal return of an individual stock. We define abnormal return as the residual of a market model in which the market return is constructed using a value-weighted portfolio comprised of all common stocks that traded on the NYSE or AMEX with valid return and volume information in the CRSP database for that day. ResVolume it, $_{i}$ is the residual volume of an individual stock. It is defined in a fashion analogous to abnormal return as the residual of a market model in which market volume is the daily detrended log turnover of the valueweighted portfolio. The table presents the results of the cross-sectional regressions:

$$
\begin{gathered}
C 2_{i}=a+b^{*} \text { ORDBA }_{i}+\text { ERROR }_{i} \\
\mathrm{C}_{2}=\mathrm{a}+\mathrm{b}^{*} \text { ORDCAP }_{\mathrm{i}}+\text { ERROR }_{\mathrm{i}}
\end{gathered}
$$

where ORDBA and ORDCAP are variables representing the ordinal scales of BAsprd and AvgCap.

T-statistics appear in parentheses.

$\mathrm{C}_{2}=\mathrm{a}+\mathrm{b}^{*} \mathrm{ORDBA}_{\mathrm{i}}+\mathrm{ERROR}_{\mathrm{i}}$

\begin{tabular}{rrrr}
\hline $\mathrm{a}$ & $\mathrm{b}$ & $\mathrm{R}^{2}(\%)$ & Observations \\
\hline 0.000740 & 0.038645 & 5.409 & 2,226 \\
$(0.374)$ & $(11.277)$ & & \\
\hline
\end{tabular}

\begin{tabular}{crrr}
$\mathrm{C}_{2} \mathrm{i}=\mathrm{a}+\mathrm{b}^{*}$ ORDCAP $_{\mathrm{i}}+$ ERROR $_{\mathrm{i}}$ & & \\
\hline $\mathrm{a}$ & $\mathrm{b}$ & $\mathrm{R}^{2}(\%)$ & Observations \\
\hline 0.033270 & -0.026386 & 2.522 & 2,226 \\
$(16.559)$ & $(-7.585)$ & & \\
& & & \\
\hline
\end{tabular}

